\title{
Subfemtometer scale color charge correlations in the proton
}

\author{
Adrian Dumitru* \\ Department of Natural Sciences, Baruch College, CUNY, \\ 17 Lexington Avenue, New York, New York 10010, USA \\ and The Graduate School and University Center, The City University of New York, \\ 365 Fifth Avenue, New York, New York 10016, USA \\ Vladimir Skokov ${ }^{\dagger}$ \\ Department of Physics, North Carolina State University, Raleigh, North Carolina 27695 \\ and RIKEN BNL Research Center, Brookhaven National Laboratory, Upton, New York 11973, USA \\ Tomasz Stebel陆 \\ Institute of Nuclear Physics PAN, Radzikowskiego 152, 31-342 Krakow, Poland \\ and Institute of Physics, Jagiellonian University, S. Lojasiewicza 11, 30-348 Krakow, Poland
}

(Received 21 January 2020; accepted 18 February 2020; published 5 March 2020)

\begin{abstract}
Color charge correlations in the proton at moderately small $x \sim 0.1$ are extracted from its light-cone wave function. The charge fluctuations are far from Gaussian and they exhibit interesting dependence on the impact parameter and on the relative transverse momentum (or distance) of the gluon probes. We provide initial conditions for small- $x$ Balitsky-Kovchegov evolution of the dipole scattering amplitude with impact parameter and $\hat{r} \cdot \hat{b}$ dependence, and with a nonzero $C$-odd component due to three-gluon exchange. Lastly, we compute the (forward) Weizsäcker-Williams gluon distributions, including the distribution of linearly polarized gluons, up to fourth order in $A^{+}$. The correction due to the quartic correlator provides a transverse momentum scale, $q \gtrsim 0.5 \mathrm{GeV}$, for nearly maximal polarization.
\end{abstract}

DOI: 10.1103/PhysRevD.101.054004

\section{INTRODUCTION}

The planned high luminosity Electron Ion Collider (EIC) is designed to perform "imaging" of the proton (and of heavy ions) with unprecedented accuracy [1]. It will provide detailed multidimensional parton distributions and insight into the light-front wave function (LFWF) of the proton via high-energy $\gamma^{(*)}-p$ scattering. The purpose of this paper is to expose the color charge correlations obtained from the LFWF of the proton.

The concept of color charge density fluctuations in the transverse impact parameter plane emerges naturally in highenergy (small- $x$ ) scattering. The projectile charge traverses without recoil the (color) field produced coherently by all "valence" charges in the target, and its propagator is given by a path ordered exponential of that field, cf. Sec. III below.

\footnotetext{
*adrian.dumitru@baruch.cuny.edu

†vskokov@ncsu.edu

*tomasz.stebel@uj.edu.pl
}

Published by the American Physical Society under the terms of the Creative Commons Attribution 4.0 International license. Further distribution of this work must maintain attribution to the author(s) and the published article's title, journal citation, and DOI. Funded by SCOAP ${ }^{3}$.
For scattering of a (virtual) photon from a proton target, this regime of coherent eikonal scattering may set in at $x \lesssim 0.1$ where the longitudinal coherence length $\sim 1 /\left(x M_{p}\right)$ of the process in the rest frame of the proton begins to exceed its radius. Nuclear targets, on the other hand, require $x \lesssim 0.1 / A^{1 / 3}$, where $A$ denotes the atomic number.

The scale separation in soft coherent fields sourced by random, "frozen" valence charges was introduced by McLerran and Venugopalan (MV) in Ref. [2]. Their model, devised for a very large nucleus, describes Gaussian fluctuations of classical color charge densities at vanishing momentum transfer: $\left\langle\rho^{a}\left(\vec{q}_{1}\right) \rho^{b}\left(\vec{q}_{2}\right)\right\rangle_{\mathrm{MV}} \sim \mu^{2} \delta\left(\vec{q}_{1}+\vec{q}_{2}\right)$. However, when the density of valence charges in the target is not very large, one would rather take the twodimensional color charge density as an operator acting on the LFWF of the target [3]. We shall see that in the regime of moderate $x \sim 0.1$ color charge fluctuations in the proton are not Gaussian, and are dependent on the impact parameter and on the transverse distance scale they are probed at.

After analyzing color charge correlations in the proton we proceed to specify initial conditions for small- $x$ Balitsky-Kovchegov (BK) evolution [4] of the dipole scattering amplitude. Detailed fits of BK evolution with running coupling corrections to the $\gamma^{*}-p$ cross section measured at Hadron-Electron Ring Accelerator (HERA) 
have been performed by Albacete et al. in Ref. [5]. More recent fits improve the accuracy of the theory by employing a collinearly improved BK evolution equation (Ref. [6] and references therein). However, such fits of small- $x$ QCD evolution to HERA deep inelastic scattering (DIS) data typically impose simplified, ad hoc initial conditions for the dipole scattering amplitude on the proton, starting at $x=10^{-2}$. We attempt to construct initial conditions based on the LFWF of the proton so that one may take advantage of "proton imaging" performed at a future EIC [1]. We use a model LFWF to show that interesting, nontrivial transverse momentum and impact parameter dependent color charge correlations in the proton should be expected. Furthermore, these initial conditions include a nonzero $C$-odd "odderon" contribution to the dipole scattering amplitude which may be evolved to smaller $x$ [7] in order to address high-energy exclusive processes involving $C$ odd exchanges or some spin dependent transverse momentum dependent (TMD) distributions such as the (dipole) gluon Sivers function of a transversely polarized proton [8].

Our final objective is to compute the WeizsäckerWilliams (WW) (forward) gluon distributions, in particular the distribution of linearly polarized gluons, at next-toleading (fourth) order in $A^{+}$(Sec. IV). At this order the conventional and linearly polarized distributions no longer coincide, and they involve the correlator of four color charge density operators in the proton. This is an independent correlation function which cannot be reduced to products of quadratic color charge correlators like in an effective theory of Gaussian color charge fluctuations. The WW gluon distribution is a TMD; its general operator definition has been provided in Refs. [9]. The WW gluon TMDs appear in a variety of processes such as production of a dijet or heavy quark pair in hadronic collisions [10] or DIS at moderate [11] or high energies [12-14], photoproduction of three jets [15], and photon pair [16], quarkonium [17], quarkonium pair [18], or quarkonium plus dilepton [19] production in hadronic collisions. These gluon distributions also determine the fluctuations of the divergence of the Chern-Simons current at the initial time of a relativistic heavy-ion collision [20].

\section{SETUP}

The light-cone (L.C.) state of an unpolarized on-shell proton with four-momentum $P^{\mu}=\left(P^{+}, P^{-}, \vec{P}_{\perp}\right)$ is written as [21]

$$
\begin{aligned}
|P\rangle= & \frac{1}{\sqrt{6}} \int \frac{\mathrm{d} x_{1} \mathrm{~d} x_{2} \mathrm{~d} x_{3}}{\sqrt{x_{1} x_{2} x_{3}}} \delta\left(1-x_{1}-x_{2}-x_{3}\right) \\
& \times \int \frac{\mathrm{d}^{2} k_{1} \mathrm{~d}^{2} k_{2} \mathrm{~d}^{2} k_{3}}{\left(16 \pi^{3}\right)^{3}} 16 \pi^{3} \delta\left(\vec{k}_{1}+\vec{k}_{2}+\vec{k}_{3}\right) \\
& \times \psi\left(x_{1}, \vec{k}_{1} ; x_{2}, \vec{k}_{2} ; x_{3}, \vec{k}_{3}\right) \sum_{i_{1}, i_{2}, i_{3}} \epsilon_{i_{1} i_{2} i_{3}}\left|p_{1}, i_{1} ; p_{2}, i_{2} ; p_{3}, i_{3}\right\rangle .
\end{aligned}
$$

The $n$-parton Fock space amplitudes are universal and process independent. They encode the nonperturbative structure of hadrons. Here, we have restricted our discussion to the valence quark Fock state, assuming that the process probes parton momentum fractions of order $x \sim 0.1$, and moderately high transverse momenta. In this regime, the above should be a reasonable first approximation.

The three on-shell quark momenta are specified by their light-cone momentum components $p_{i}^{+}=x_{i} P^{+}$and their transverse components $\vec{p}_{i}=x_{i} \vec{P}_{\perp}+\vec{k}_{i}$. The colors of the quarks are denoted by $i_{1,2,3}$. We omit helicity quantum numbers (and flavor indices) as they play no role in our analysis. $\psi$ is symmetric under exchange of any two of the quarks, and is normalized according to

$$
\begin{aligned}
& \int \mathrm{d} x_{1} \mathrm{~d} x_{2} \mathrm{~d} x_{3} \delta\left(1-x_{1}-x_{2}-x_{3}\right) \\
& \times \int \frac{\mathrm{d}^{2} k_{1} \mathrm{~d}^{2} k_{2} \mathrm{~d}^{2} k_{3}}{\left(16 \pi^{3}\right)^{3}}\left(16 \pi^{3}\right) \delta\left(\vec{k}_{1}+\vec{k}_{2}+\vec{k}_{3}\right)|\psi|^{2}=1 .
\end{aligned}
$$

This corresponds to the proton state normalization

$$
\langle K \mid P\rangle=16 \pi^{3} P^{+} \delta\left(P^{+}-K^{+}\right) \delta\left(\vec{P}_{\perp}-\vec{K}_{\perp}\right) .
$$

Below, we neglect plus momentum transfer so that $\xi=\left(K^{+}-P^{+}\right) / P^{+} \rightarrow 0$. This approximation is valid at high energies.

For numerical estimates we employ a model wave function $\psi\left(x_{1}, \vec{k}_{1} ; x_{2}, \vec{k}_{2} ; x_{3}, \vec{k}_{3}\right)$ described in the Appendix A.

\section{DIPOLE SCATTERING AMPLITUDE}

The $S$-matrix for scattering of a quark-antiquark dipole off the fields in the target proton can be expressed as (see, e.g., Ref. [22])

$$
\mathcal{S}(\vec{r}, \vec{b})=\frac{1}{N_{c}} \operatorname{tr}\left\langle U\left(\vec{b}+\frac{\vec{r}}{2}\right) U^{\dagger}\left(\vec{b}-\frac{\vec{r}}{2}\right)\right\rangle .
$$

Following the standard convention in the small- $x$ literature we define the scattering amplitude

$$
\mathcal{T}(\vec{r}, \vec{b})=1-\mathcal{S}(\vec{r}, \vec{b}),
$$

without a factor of $i$.

When integrated over impact parameters $\vec{b}$, Eq. (5) is related to the so-called dipole gluon distribution [23]. Here, $U\left(U^{\dagger}\right)$ are (anti)path ordered Wilson lines representing the eikonal scattering of the dipole of size $\vec{r}$ at impact parameter $\vec{b}$ :

$$
\begin{aligned}
U\left(\vec{x}_{T}\right) & =\mathcal{P} e^{i g \int d x^{-} A^{+a}\left(x^{-}, \vec{x}_{T}\right) t^{a}}, \\
U^{\dagger}\left(\vec{x}_{T}\right) & =\overline{\mathcal{P}} e^{-i g \int d x^{-} A^{+a}\left(x^{-}, \vec{x}_{T}\right) t^{a}} .
\end{aligned}
$$



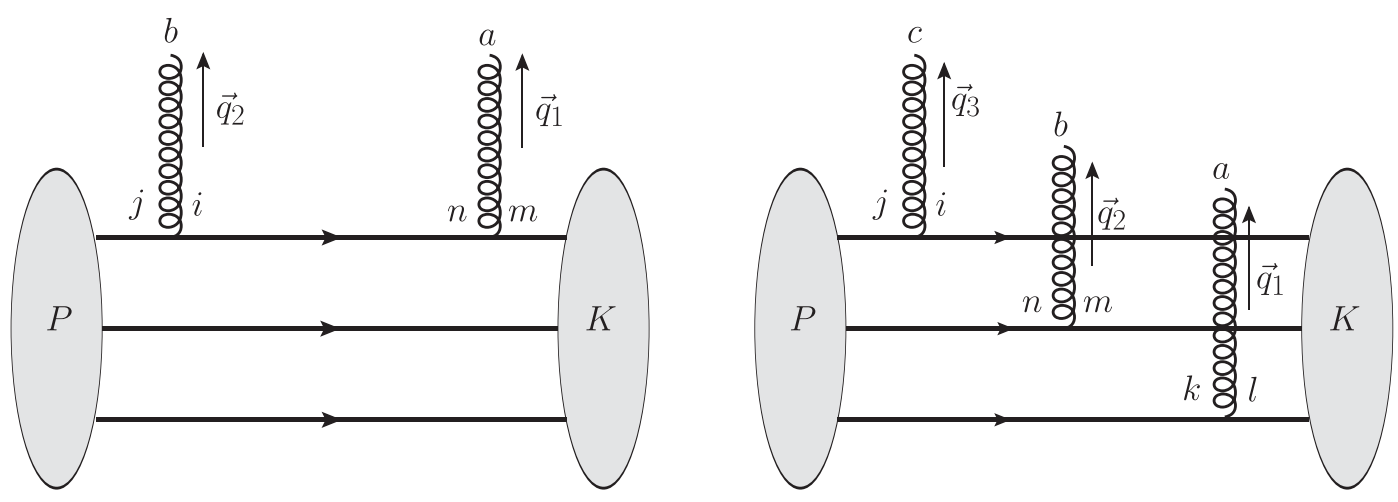

FIG. 1. Left: one of the diagrams for the correlator $\left\langle\rho^{a}\left(\vec{q}_{1}\right) \rho^{b}\left(\vec{q}_{2}\right)\right\rangle$ (once Coulomb propagators are amputated); this contribution dominates at large relative gluon momenta but small total momentum transfer $\vec{K}_{T}=-\vec{q}_{1}-\vec{q}_{2}$. Right: one of the diagrams for the correlator $\left\langle\rho^{a}\left(\vec{q}_{1}\right) \rho^{b}\left(\vec{q}_{2}\right) \rho^{c}\left(\vec{q}_{3}\right)\right\rangle$; this contribution dominates when the three gluons share a large momentum transfer, $\vec{K}_{T} / 3 \simeq-\vec{q}_{1} \simeq-\vec{q}_{2} \simeq-\vec{q}_{3}$.

$\mathcal{S}(\vec{r}, \vec{b})$ and $\mathcal{T}(\vec{r}, \vec{b})$ are invariant under the simultaneous $\mathcal{P} \leftrightarrow \overline{\mathcal{P}}, \vec{r} \rightarrow-\vec{r}, g A^{+} \rightarrow-g A^{+}$. We now expand $\mathcal{T}(\vec{r}, \vec{b})$ to third order in $g A^{+}$, neglecting exchanges of more than three gluons, and write it in terms of correlators of the field integrated over the longitudinal coordinate:

$$
\begin{aligned}
& A^{+a}\left(\vec{x}_{T}\right)=\int \mathrm{d} x^{-} A^{+a}\left(\vec{x}_{T}, x^{-}\right), \\
& A^{+a}\left(\vec{x}_{T}\right) A^{+b}\left(\vec{y}_{T}\right)+A^{+b}\left(\vec{y}_{T}\right) A^{+a}\left(\vec{x}_{T}\right) \\
& \quad=\mathcal{P} \int \mathrm{d} x^{-} \int \mathrm{d} y^{-} A^{+a}\left(\vec{x}_{T}, x^{-}\right) A^{+b}\left(\vec{y}_{T}, y^{-}\right) \\
& \quad+\overline{\mathcal{P}} \int \mathrm{d} x^{-} \int \mathrm{d} y^{-} A^{+a}\left(\vec{x}_{T}, x^{-}\right) A^{+b}\left(\vec{y}_{T}, y^{-}\right) .
\end{aligned}
$$

This field is related to the $2 \mathrm{~d}$ color charge density through

$$
-\nabla_{\perp}^{2} A^{+a}\left(\vec{x}_{T}\right)=\rho^{a}\left(\vec{x}_{T}\right),
$$

allowing us to express the dipole scattering amplitude in terms of color charge density correlators. Some of the diagrams that contribute to the two- and three-gluon exchange amplitudes are shown in Fig. 1. The general relation of correlators of Wilson lines at small $x$ to generalized parton distributions has been elucidated in Ref. [24], to all twists.

$C$-even two gluon exchange corresponds to the scattering amplitude [3]

$$
\begin{aligned}
\mathcal{T}_{g g}(\vec{r}, \vec{b})= & -\frac{g^{4}}{2} C_{F} \int_{K_{T}, q} \frac{e^{-i \vec{b} \cdot \vec{K}_{T}}}{\left(\vec{q}-\frac{1}{2} \vec{K}_{T}\right)^{2}\left(\vec{q}+\frac{1}{2} \vec{K}_{T}\right)^{2}} \\
& \times\left(\cos (\vec{r} \cdot \vec{q})-\cos \left(\frac{\vec{r} \cdot \vec{K}_{T}}{2}\right)\right) \\
& \times G_{2}\left(\vec{q}-\frac{1}{2} \vec{K}_{T},-\vec{q}-\frac{1}{2} \vec{K}_{T}\right) .
\end{aligned}
$$

[We use the shorthand notation $\int_{q}=\int \mathrm{d}^{2} q /(2 \pi)^{2}$.] Here, we introduced the color charge correlator

$$
\left\langle\rho^{a}\left(\vec{q}_{1}\right) \rho^{b}\left(\vec{q}_{2}\right)\right\rangle \equiv \operatorname{tr} t^{a} t^{b} g^{2} G_{2}\left(\vec{q}_{1}, \vec{q}_{2}\right) ;
$$

see Appendix B for details. It is symmetric under a simultaneous sign flip of both arguments and so $\mathcal{T}_{g g}(\vec{r}, \vec{b})$ is real. The integral in Eq. (9) is free of infrared divergences since $G_{2}$ satisfies a Ward identity and vanishes when either one of the gluon momenta goes to zero [25,26]: $G_{2}\left(\vec{q}-\frac{1}{2} \vec{K}_{T},-\vec{q}-\frac{1}{2} \vec{K}_{T}\right) \sim\left(\vec{q} \pm \frac{1}{2} \vec{K}_{T}\right)^{2} \quad$ as $\quad \vec{q} \rightarrow \pm \frac{1}{2} \vec{K}_{T}$. In Fig. 2 we show a numerical estimate for $G_{2}$ as a function of impact parameter $b$ or relative momentum $\vec{q}_{12}=\vec{q}_{1}-\vec{q}_{2}=2 \vec{q}_{1}+\vec{K}_{T}$ :

$\tilde{G}_{2}\left(\vec{q}_{12}, \vec{b}\right)=\int_{K_{T}} e^{-i \vec{b} \cdot \vec{K}_{T}} G_{2}\left(\frac{\vec{q}_{12}-\vec{K}_{T}}{2},-\frac{\vec{q}_{12}+\vec{K}_{T}}{2}\right)$.

We also average over the relative directions of $\vec{q}_{12}$ and $\vec{b}$. For numerical estimates we used the model wave function by Brodsky and Schlumpf [27] described briefly in Appendix A.

$G_{2}$ measures charge correlations seen by two gluon probes of the same color. There is a color charge anticorrelation ("repulsion") at small relative momentum of the gluon probes in the center of the proton which turns into a positive correlation ("attraction") towards the periphery, or at high relative momentum. The integral of $\tilde{G}_{2}$ over the $2 \mathrm{~d}$ impact parameter plane at vanishing relative momentum is zero:

$$
\int \mathrm{d}^{2} b \tilde{G}_{2}\left(\vec{q}_{12}=0, \vec{b}\right)=0
$$

A similar relation holds for the cubic charge correlators discussed below. 

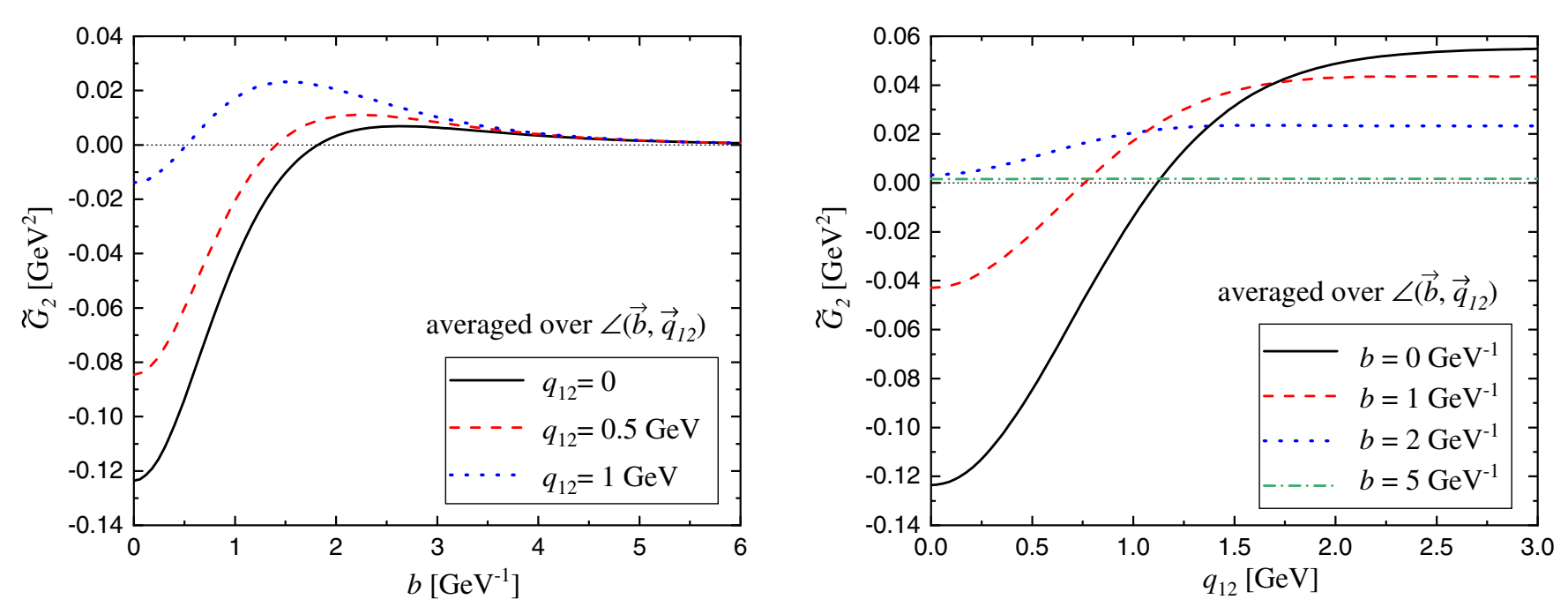

FIG. 2. The quadratic color charge density correlator $\tilde{G}_{2}\left(\vec{q}_{12}, b\right)$ in the proton as a function of impact parameter and relative transverse momentum of the two gluon probes.

At third order in $A^{+a}$ we have the following scattering amplitude for $C$-odd three gluon exchange [3]:

$$
\begin{aligned}
\mathcal{T}_{g g g}(\vec{r}, \vec{b})= & \frac{5}{18} g^{6} \int_{q_{1}, q_{2}, q_{3}} \frac{1}{q_{1}^{2}} \frac{1}{q_{2}^{2}} \frac{1}{q_{3}^{2}} e^{-i \vec{b} \cdot \vec{K}_{T}} G_{3}^{-}\left(\vec{q}_{1}, \vec{q}_{2}, \vec{q}_{3}\right) \\
& \times\left[\sin \left(\vec{r} \cdot \vec{q}_{1}+\frac{1}{2} \vec{r} \cdot \vec{K}_{T}\right)-\frac{1}{3} \sin \left(\frac{1}{2} \vec{r} \cdot \vec{K}_{T}\right)\right] .
\end{aligned}
$$

Here, $\vec{K}_{T} \equiv-\left(\vec{q}_{1}+\vec{q}_{2}+\vec{q}_{3}\right)$. We denote the $C$-odd part of the correlator of three color charges as

$$
\left\langle\rho^{a}\left(\vec{q}_{1}\right) \rho^{b}\left(\vec{q}_{2}\right) \rho^{c}\left(\vec{q}_{3}\right)\right\rangle_{C=-} \equiv \frac{1}{4} d^{a b c} g^{3} G_{3}^{-}\left(\vec{q}_{1}, \vec{q}_{2}, \vec{q}_{3}\right)
$$

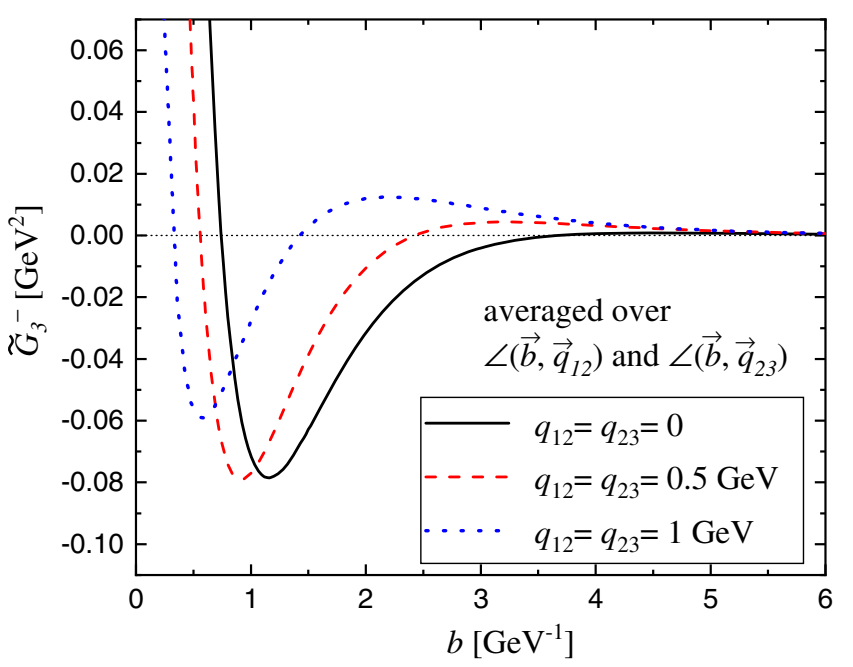

This correlator, too, is symmetric under a simultaneous sign flip of all three gluon momenta and so $\mathcal{T}_{g g g}(\vec{r}, \vec{b})$ is imaginary. Also, it vanishes quadratically in any of the transverse momentum arguments so that $\mathcal{T}_{\text {ggg }}(\vec{r}, \vec{b})$ is free of infrared divergences.

The fact that $G_{3}^{-}$does not vanish shows that color charge fluctuations in the proton state (1) are not Gaussian. A numerical estimate of $\tilde{G}_{3}^{-}$is shown in Fig. 3. At small relative momentum we observe a positive correlation at the center of the proton; $\tilde{G}_{3}^{-}(b)$ diverges logarithmically at $b \rightarrow 0$ due to contributions from large momentum transfer $-t=K_{T}^{2}$. This turns into an anticorrelation around $b \approx 1 \mathrm{GeV}^{-1}$, and then vanishes for large impact parameters. At high relative momentum the correlator is large and

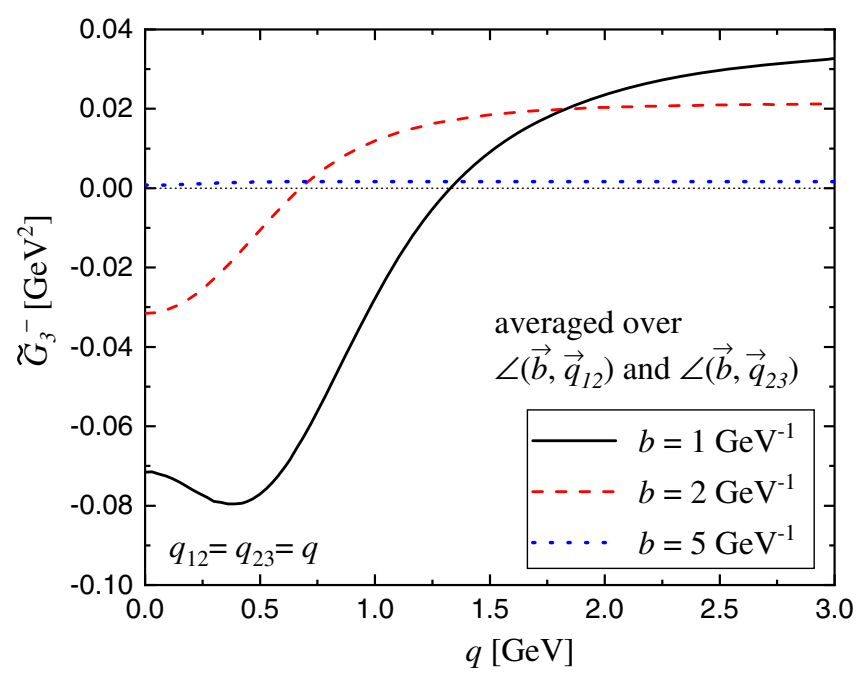

FIG. 3. The $C$-odd part of the cubic color charge density correlator $\tilde{G}_{3}^{-}$in the proton as a function of impact parameter and relative transverse momentum. 

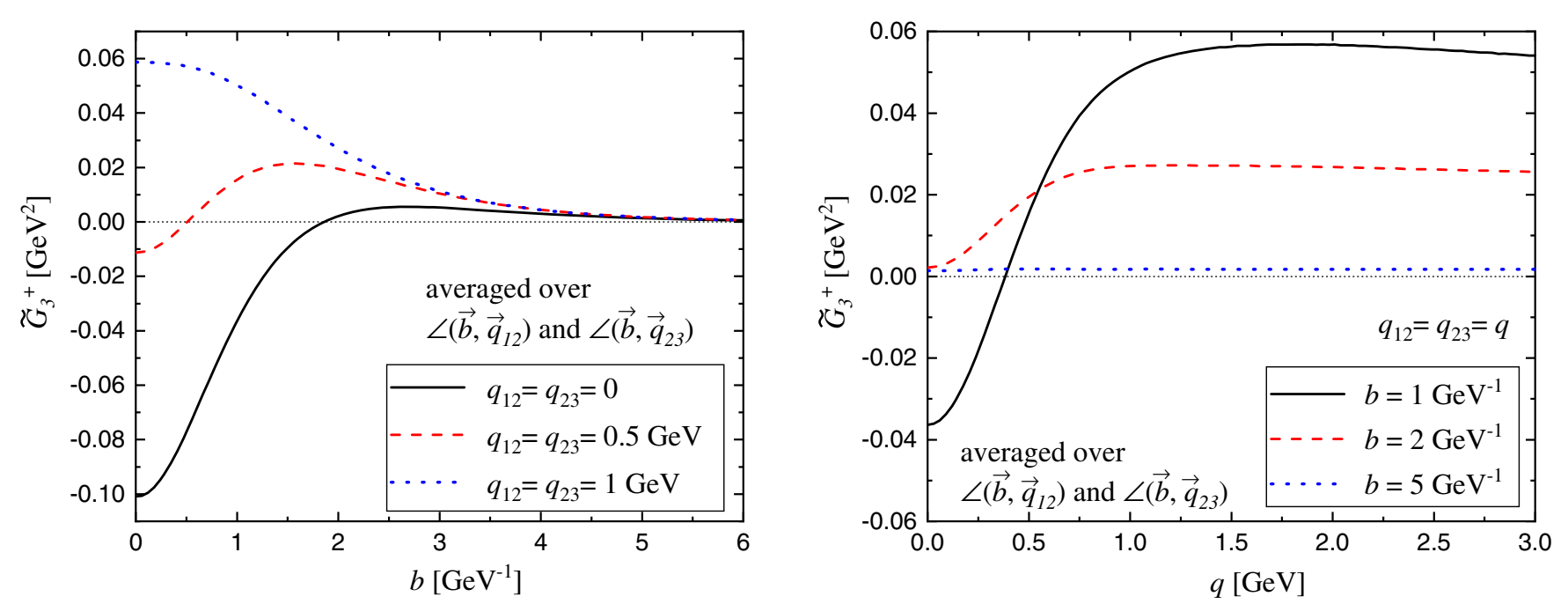

FIG. 4. The $C$-even part of the cubic color charge density correlator $\tilde{G}_{3}^{+}$in the proton as a function of impact parameter and relative transverse momentum.

positive at small $b$. For generic impact parameters and momenta $\tilde{G}_{2}$ and $\tilde{G}_{3}^{-}$are of similar numerical magnitude.

For completeness, we finally show the $C$-even part of the correlator of three color charges,

$$
\left\langle\rho^{a}\left(\vec{q}_{1}\right) \rho^{b}\left(\vec{q}_{2}\right) \rho^{c}\left(\vec{q}_{3}\right)\right\rangle_{C=+} \equiv \frac{i}{4} f^{a b c} g^{3} G_{3}^{+}\left(\vec{q}_{1}, \vec{q}_{2}, \vec{q}_{3}\right)
$$

even though it does not contribute to the dipole scattering amplitude. This correlator is negative near the center, and for small relative momenta, then turns into a positive correlation at large momenta, c.f. Fig. 4.

All three color charge correlators decay with increasing impact parameter, just as expected intuitively. Observing the correlations at small $b$ involves large momentum transfer to the proton to zoom in on its center. The regime where the exchanged gluons share a large momentum transfer $-t=K_{T}^{2}$ is dominated by $n$-body diagrams such as the one shown in Fig. 1(right), where the static gluons attach to as many sources as possible ${ }^{1}$ [29]. This leads to the greatest overlap of the wave functions of incoming and scattered protons.

We now show the behavior of the dipole scattering amplitude $\mathcal{T}(\vec{b}, \vec{r})$. For all figures we assumed a fixed $\alpha_{s}=0.35[29,30]$ and we align the impact parameter and dipole vectors. However, the scattering amplitude does depend on the relative orientation of $\vec{b}$ and $\vec{r}^{2}$

The two gluon exchange amplitude $\mathcal{T}_{g g}(\vec{b}, \vec{r})$ is shown in Fig. 5. It displays the expected roughly exponential falloff

\footnotetext{
${ }^{1}$ This was first noted by Donnachie and Landshoff who argued that three gluon exchange should dominate over two-gluon exchange in elastic proton-proton scattering at high energy and large $-t(\ll s)$ [28].

${ }^{2}$ This would give rise to azimuthal correlations in double parton scattering in hadronic collisions [31].
}

at large impact parameters. The amplitude is significantly smaller than 1 even at the center of the proton, albeit not by several orders of magnitude, e.g., $\mathcal{T}_{g q} \simeq 0.1$ at $b=$ $1 \mathrm{GeV}^{-1}$ and $r=2 \mathrm{GeV}^{-1}$. Matching this to $\mathcal{T}_{g g}=$ $\frac{1}{4} r^{2} Q_{s}^{2}(b)$ would correspond to a saturation momentum of about $Q_{s}(b) \approx 0.3 \mathrm{GeV}$ at $b=1 \mathrm{GeV}^{-1}$ and $x \sim 0.1$. For comparison, we recall $Q_{s} \approx 0.4-0.5 \mathrm{GeV}$ at $x=0.01$, on average over impact parameters, extracted from systematic fits of BK evolution with running coupling corrections to HERA data for $F_{2}$ [5].

As expected, $\mathcal{T}_{g g}(\vec{r})$ at fixed $b$ first increases with the size of the dipole; the slope is less steep at larger impact parameters where the target is more "dilute." The scattering amplitude eventually reaches a maximum value for $r_{\max } \gtrsim 5 \mathrm{GeV}^{-1}$ beyond which it decreases again as the projectile dipole "misses" the target. ${ }^{3}$ However, this behavior occurs in a regime of large dipoles where the analysis of the scattering amplitude (and of $\gamma^{(*)} \rightarrow q \bar{q}$ ) in perturbation theory is not valid.

The $C$-odd three gluon exchange amplitude (odderon ${ }^{4}$ ) $-i \mathcal{T}_{\text {ggg }}(\vec{b}, \vec{r})$ is shown in Fig. 6. This amplitude changes sign under $\vec{b} \rightarrow-\vec{b}$ (negative parity) and vanishes at $b=0$. Its magnitude is maximal at $b \sim 0.5-1.2 \mathrm{GeV}^{-1}$, approximately where the gradient of the two-gluon exchange amplitude is greatest [34]. For impact parameters $b \lesssim$ $3 \mathrm{GeV}^{-1}$ and small dipoles, $r \lesssim 4 \mathrm{GeV}^{-1}$, we find that $\mathcal{T}_{g g g}$ is smaller than $\mathcal{T}_{g g}$ by at least one order of

\footnotetext{
${ }^{3}$ This behavior also emerges as a consequence of impact parameter dependent small- $x$ BK evolution, even when the dipole amplitude at the initial $x_{0}$ increases monotonically with $r$ [32].

${ }^{4}$ We should mention that we restrict our discussion to the odderon associated with (relatively large) transverse momentum transfer $\vec{K}_{T}$. For nearly forward scattering another odderon exchange associated with a spin flip of the proton may appear [33].
} 

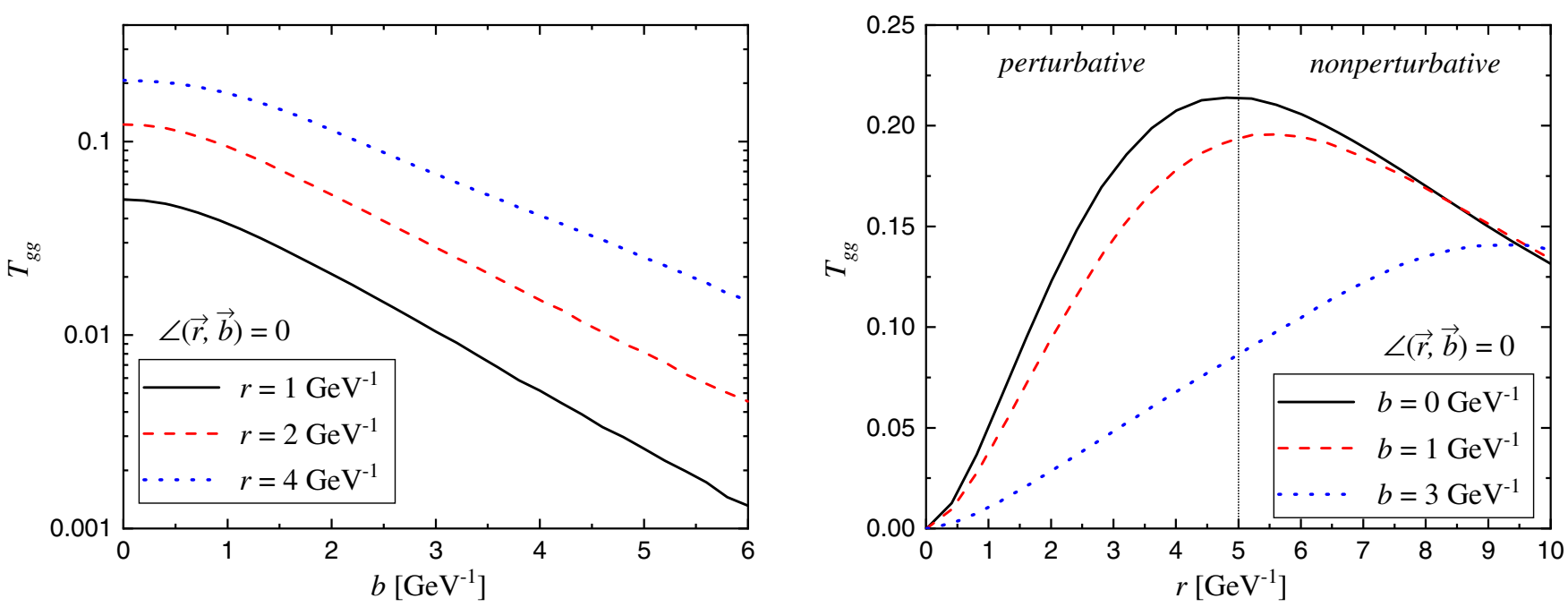

FIG. 5. The two gluon exchange amplitude $\mathcal{T}_{g g}(\vec{b}, \vec{r})$.
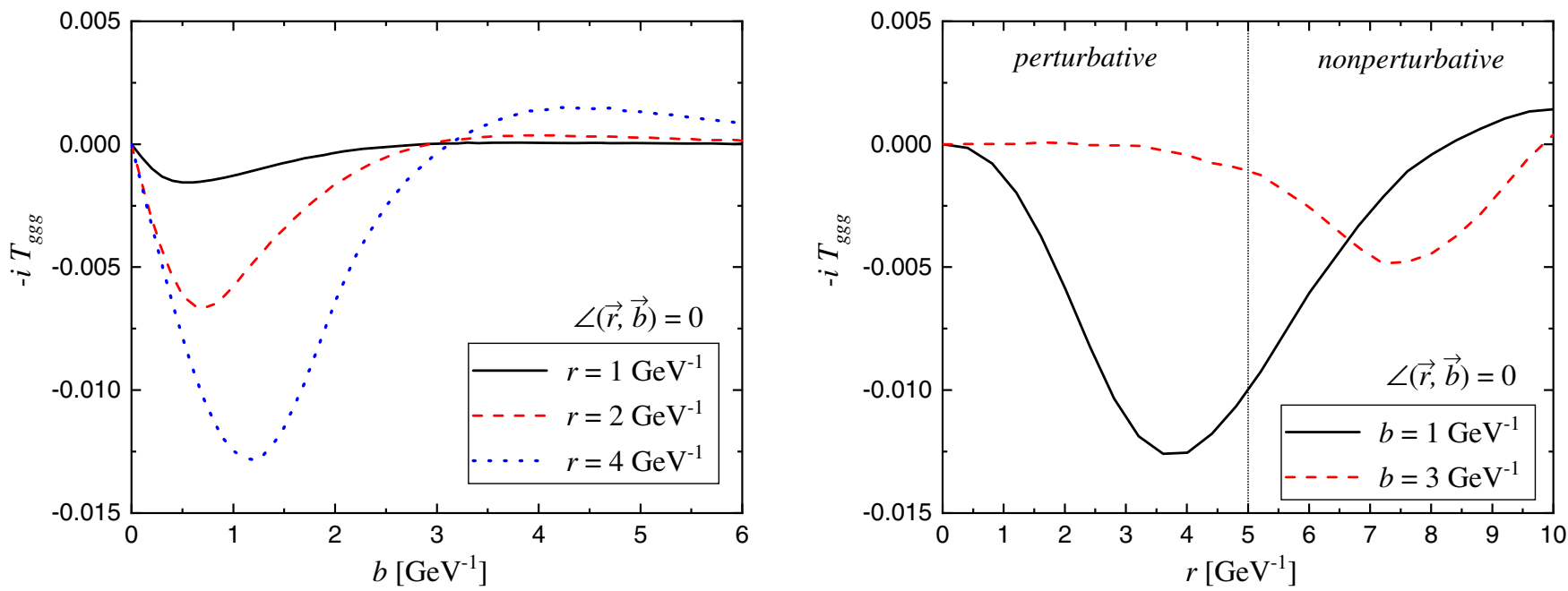

FIG. 6. The $C$-odd three gluon exchange amplitude $\operatorname{Im} \mathcal{T}_{\text {ggg }}(\vec{b}, \vec{r})$.

magnitude. ${ }^{5}$ This is not because color charge fluctuations in the proton are nearly Gaussian, as the magnitudes of $G_{2}$ and $G_{3}^{-}$(shown above) are similar. Rather, it appears to originate mostly from the parity odd nature of $\mathcal{T}_{\text {ggg }}$ which gives rise to large cancellations in the integral in Eq. (13). As a consequence, semihard processes requiring $C$-odd three gluon exchange have small cross sections [29]. Alternatively, one may search for the perturbative odderon via charge asymmetries in diffractive electroproduction of a $\pi^{+} \pi^{-}$pair [35].

\footnotetext{
${ }^{5}$ The magnitude of $\operatorname{Im} \mathcal{T}_{\text {ggg }}$ obtained from the present LFWF is one order of magnitude smaller than the one used as the initial condition for small- $x$ evolution in Ref. [8], where the authors compute the dipole gluon Sivers function in a transversely polarized proton.
}

\section{WEIZSÄCKER-WILLIAMS GLUON DISTRIBUTIONS}

In this section we relate the color charge correlators to the (forward) WW gluon distribution. It is given, at small- $x$, by the correlator of two light-cone gauge fields $[23,36]$

$$
\begin{aligned}
x G_{\mathrm{WW}}^{i j}(x, \vec{q})= & \frac{1}{2} \delta^{i j} x G^{(1)}(x, \vec{q}) \\
& +\frac{1}{2}\left(2 \frac{q^{i} q^{j}}{q^{2}}-\delta^{i j}\right) x h_{\perp}^{(1)}(x, \vec{q}) \\
= & \frac{1}{4 \pi^{3}}\left\langle A^{i a}(\vec{q}) A^{j a}(-\vec{q})\right\rangle .
\end{aligned}
$$

The trace of $x G_{\mathrm{WW}}^{i j}$ defines the conventional WW gluon distribution $x G^{(1)}(x, \vec{q})$ while the traceless part corresponds to the distribution of linearly polarized gluons $x h_{\perp}^{(1)}(x, \vec{q})$. 

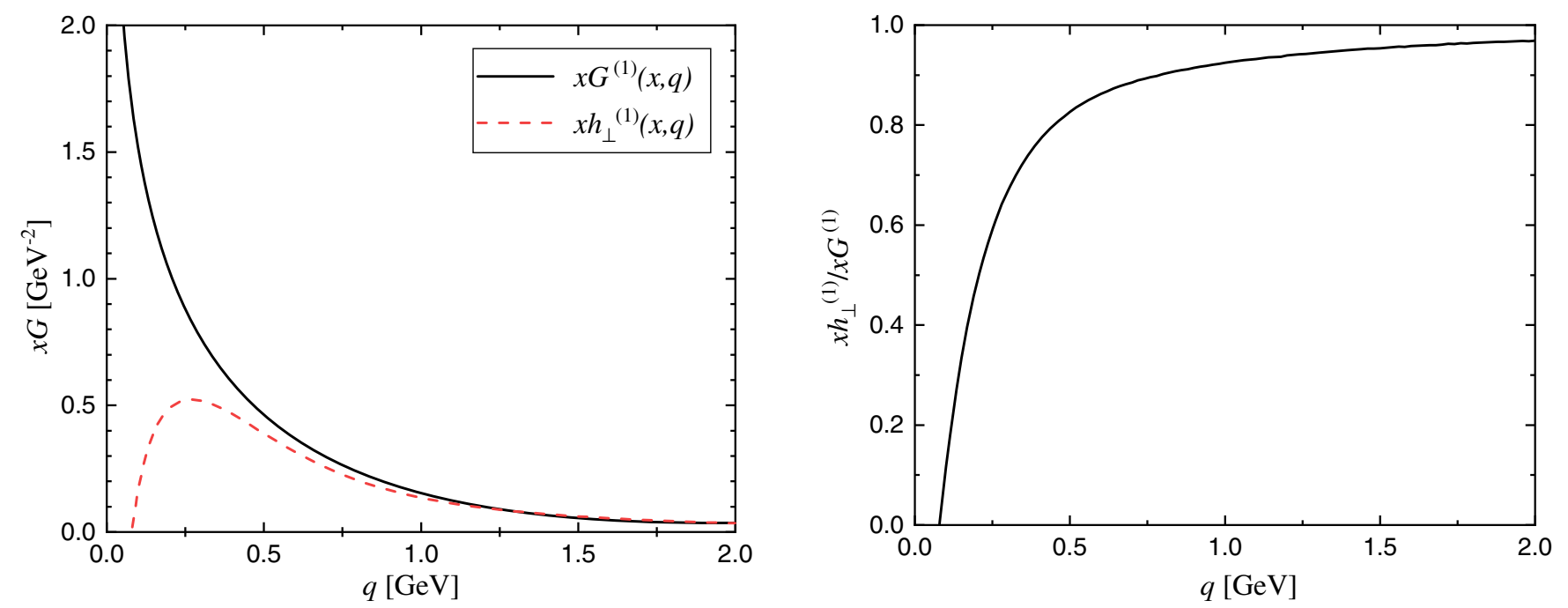

FIG. 7. The conventional and linearly polarized WW gluon distributions in the proton (at $x \sim 0.1)$ to order $\left(A^{+}\right)^{4}$.

Both are integrated over impact parameters since we consider the forward limit. In the nonforward case the general decomposition of the WW generalized transverse momentum dependent distribution (GTMD) involves additional independent functions on the right-hand side of Eq. (16); see, e.g., Ref. [37].

The field in light-cone gauge is obtained from $A^{+}$by a gauge transformation,

$$
A^{i}\left(\vec{x}_{T}\right)=\frac{i}{g} U^{\dagger}\left(\vec{x}_{T}\right) \partial^{i} U\left(\vec{x}_{T}\right)
$$

such that in this gauge $A^{+}\left(\vec{x}_{T}\right)=0$. At linear order in $\rho, A^{i}(\vec{q}) \sim q^{i} \rho(\vec{q})$ is longitudinal so that $x G^{(1)}(x, \vec{q})=$ $x h_{\perp}^{(1)}(x, \vec{q})$, corresponding to maximal polarization:

$$
x G^{(1)}(x, \vec{q})=x h_{\perp}^{(1)}(x, \vec{q})=\frac{N_{c}^{2}-1}{8 \pi^{3} q^{2}} g^{2} G_{2}(\vec{q},-\vec{q}) .
$$

Beyond leading order in $\rho$ (or $A^{+}$) the L.C. gauge field is no longer purely longitudinal and one finds that $x G^{(1)}(x, \vec{q})>x h_{\perp}^{(1)}(x, \vec{q})$. See Refs. [13,38] for computations of these distributions to all orders in $A^{+}$, in the Gaussian MV model of classical color charges. Resummed WW gluon distributions for Gaussian color charge fluctuations with a more general two-point correlator have been derived in Ref. [39]; also see Appendix C.

Here, we express the correction to $x G^{(1)}(x, \vec{q})$ and $x h_{\perp}^{(1)}(x, \vec{q})$ at fourth order in $A^{+}$in terms of the quartic color charge correlator:

$$
\begin{aligned}
\Delta x G^{(1)}(x, \vec{q})=-\Delta x h_{\perp}^{(1)}(x, \vec{q})= & \frac{g^{2}}{16 \pi^{3}} f^{a b e} f^{c d e} \int_{k, p} \frac{1}{k^{2}} \frac{1}{p^{2}} \frac{1}{(\vec{q}-\vec{k})^{2}} \frac{1}{(\vec{q}+\vec{p})^{2}}\left(\frac{\vec{k} \cdot \vec{q} \vec{p} \cdot \vec{q}}{q^{2}}-\vec{k} \cdot \vec{p}\right) \\
& \times\left\langle\rho^{a}(\vec{q}-\vec{k}) \rho^{b}(\vec{k}) \rho^{c}(-\vec{q}-\vec{p}) \rho^{d}(\vec{p})\right\rangle .
\end{aligned}
$$

The explicit expression for $f^{a b e} f^{c d e}\left\langle\rho^{a}(\vec{q}-\vec{k}) \rho^{b}(\vec{k})\right.$ $\left.\rho^{c}(-\vec{q}-\vec{p}) \rho^{d}(\vec{p})\right\rangle$ in terms of the proton LFWF is given in Eq. (C7) of Appendix C. Hence, at this order there is a splitting of $x G^{(1)}$ and $x h_{\perp}^{(1)}$ which are no longer equal.

Figure 7 shows numerical results for the two WW gluon distributions in the proton. For $q \gtrsim 0.5 \mathrm{GeV}$ the higher twist correction is very small and the "polarization" is nearly maximal. This confirms that a measurement of $x h_{\perp}^{(1)}(x, q)$ at an EIC appears promising, for example via dijet azimuthal asymmetries [12]. The higher twist correction overwhelms the leading contribution below $q \sim$ $0.2 \mathrm{GeV}$ where a resummation to all orders in $A^{+}$would be required. For the Gaussian MV model of classical color charge fluctuations this has been done in Refs. [13,38] (and its evolution to small $x$ in Refs. $[14,40]$ ) but here higher order correlators are independent functions and a resummation appears difficult.

\section{SUMMARY AND DISCUSSION}

In this paper we have computed $2 \mathrm{~d}$ color charge density correlations in the proton at moderate $x \sim 0.1$. The correlators of two, three, and four color charge density operators $\rho^{a}$ have been related explicitly to the light-front wave function of the proton. These correlators exhibit interesting dependence on the relative momenta of the probes, and on 
impact parameter. The two-point correlator $G_{2}\left(\vec{q}_{1}, \vec{q}_{2}\right) \sim$ $\left\langle\rho^{a}\left(\vec{q}_{1}\right) \rho^{a}\left(\vec{q}_{2}\right)\right\rangle$, for example, is positive at large relative momentum $\vec{q}_{12}=\vec{q}_{1}-\vec{q}_{2}$, indicating "attraction" of like charges. It turns negative ("repulsion") at smaller relative momentum, for central impact parameters. The correlation function satisfies a sum rule such that at $q_{12}=0$ its integral over the impact parameter plane vanishes: $\int \mathrm{d}^{2} b \tilde{G}_{2}\left(\vec{b}, q_{12}=0\right)=0$. We note that $\tilde{G}_{2}\left(\vec{b}, \vec{q}_{12}\right)$ is a two-body generalized parton distribution (GPD) which depends not only on impact parameter but also on the relative transverse momentum (or distance) of the two gluon probes ${ }^{6}$ :

$$
\begin{aligned}
\tilde{G}_{2}\left(\vec{b}, \vec{q}_{12}\right)= & \int_{K_{T}} e^{-i \vec{b} \cdot \vec{K}_{T}} \int \mathrm{d} x_{1} \mathrm{~d} x_{2} \mathrm{~d} x_{3} \delta\left(1-x_{1}-x_{2}-x_{3}\right) \int \frac{\mathrm{d}^{2} p_{1} \mathrm{~d}^{2} p_{2} \mathrm{~d}^{2} p_{3}}{\left(16 \pi^{3}\right)^{2}} \delta\left(\vec{p}_{1}+\vec{p}_{2}+\vec{p}_{3}\right) \\
& \times\left[\psi^{*}\left(\vec{p}_{1}+\left(1-x_{1}\right) \vec{K}_{T}, \vec{p}_{2}-x_{2} \vec{K}_{T}, \vec{p}_{3}-x_{3} \vec{K}_{T}\right)\right. \\
& \left.-\psi^{*}\left(\vec{p}_{1}-\frac{\vec{q}_{12}-\vec{K}_{T}}{2}-x_{1} \vec{K}_{T}, \vec{p}_{2}+\frac{\vec{q}_{12}+\vec{K}_{T}}{2}-x_{2} \vec{K}_{T}, \vec{p}_{3}-x_{3} \vec{K}_{T}\right)\right] \psi\left(\vec{p}_{1}, \vec{p}_{2}, \vec{p}_{3}\right) .
\end{aligned}
$$

$\psi$ denotes the amplitude of the three-quark Fock state of the proton. The first, one-body term is dominant for large $b$ and $q_{12}$ while the second, two-body contribution dominates for small $b$ and $q_{12}$. To illustrate the importance of $n$-body contributions to the color charge correlators, in Fig. 8 we compare $\tilde{G}_{2}\left(\vec{b}, q_{12}=0\right)$ and $\tilde{G}_{3}^{-}\left(\vec{b}, q_{12}=q_{23}=0\right)$ to the one-body quark density ${ }^{7}$ in impact parameter space, i.e., to the proton "thickness function" $T_{p}(b)$. Even at vanishing relative momenta these coincide only at rather large $b$. The color charge correlators $\left\langle\rho^{a}\left(\vec{q}_{1}\right) \rho^{b}\left(\vec{q}_{2}\right)\right\rangle$ and $\left\langle\rho^{a}\left(\vec{q}_{1}\right) \rho^{b}\left(\vec{q}_{2}\right) \rho^{c}\left(\vec{q}_{3}\right)\right\rangle_{C=-}$ can be probed in exclusive production of various charmonium states in (virtual) photonproton scattering $[29,42]$ or via charge asymmetries in pion pair production [35].

Another main result of the paper is that color charge fluctuations in the proton are far from Gaussian. The magnitudes of the $C$-even and $C$-odd components of the cubic correlator $\left\langle\rho^{a} \rho^{b} \rho^{c}\right\rangle / g^{3}$ are comparable to that of the two-point correlator $\left\langle\rho^{a} \rho^{b}\right\rangle / g^{2}$. In particular, $C$-odd correlations of cubic fluctuations near the center of the proton are large and positive, for sufficiently small relative momenta of the gluon probes.

Subfemto-scale color charge correlations in the proton determine the dipole scattering amplitude. Relating them to the proton LFWF, which could in principle be determined via "imaging" of the proton at a future electron-ion collider, could help constrain and improve initial conditions for small- $x$ evolution. In particular, our analysis provides initial conditions which account for the above-mentioned nontrivial structure of two- and three-point correlators as functions of the transverse momentum $\left(\vec{q}_{12}\right)$ or distance scale $(\vec{r})$, impact parameter $\vec{b}$, and their relative angular

\footnotetext{
${ }^{6}$ For the proton wave function considered here, there is no dependence on $x$. We refer to Ref. [41] for a review on GPDs.

${ }^{7}$ The quark density is given by three times the first term in Eq. (20).
}

orientation. Hence, they may be useful for checking the consistency of BK evolution with the impact parameter dependence of the dipole $S$-matrix extracted from data at small $x$ [43].

The scattering amplitude derived here also includes a nonzero $C$-odd odderon contribution to the dipole scattering amplitude which may be evolved to smaller $x$ [7] to predict cross sections for exclusive processes involving $C$-odd exchanges, or the dipole gluon Sivers function of a transversely polarized proton [8]. Somewhat surprisingly, perhaps, our numerical analysis indicates that the $C$-odd amplitude for three-gluon exchange $\mathcal{T}_{\text {ggg }}(\vec{r}, \vec{b})$ is much smaller in magnitude than the $C$-even amplitude $\mathcal{T}_{g g}(\vec{r}, \vec{b})$ for two-gluon exchange. As already mentioned, this is not because color charge fluctuations in the proton are nearly Gaussian. Neither is it due to the additional power of $\alpha_{s}$ in $\mathcal{T}_{\text {ggg }}(\vec{r}, \vec{b})$ which is compensated by other numerical

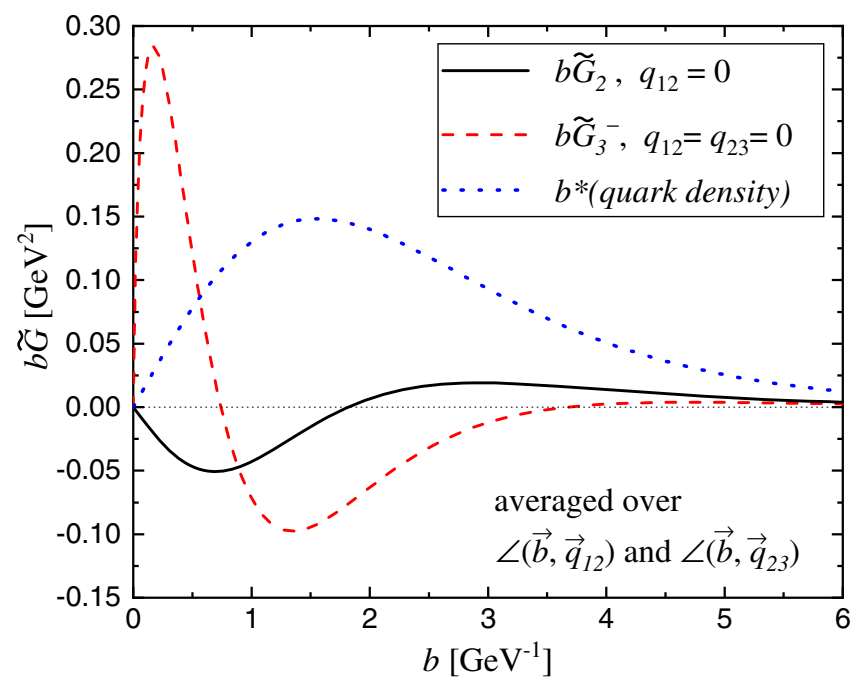

FIG. 8. Quadratic and C-odd cubic color charge correlators, and the one-body quark density, as functions of impact parameter. 
factors. Rather, it is mainly a consequence of the fact that this amplitude is odd under parity. This leads to large cancellations in the three gluon exchange diagram (for central impact parameters) when their transverse momenta are reversed. $\mathcal{T}_{g g g}(\vec{r}, \vec{b})$ must vanish, also, for large impact parameters or large dipoles as the density of color charge if the periphery of the proton is low. Consequently, we expect that cross sections for semihard exclusive processes involving $C$-odd three-gluon exchange are small and require high luminosity.

We have also computed the conventional and linearly polarized Weizsäcker-Williams gluon TMDs $x G^{(1)}(x, q)$ and $x h_{\perp}^{(1)}(x, q)$ in the proton at moderately low $x \sim 0.1$. At leading twist [order $\left(A^{+}\right)^{2}$ ] the field in light-cone gauge is purely longitudinal and there is maximal polarization, $x G^{(1)}(x, q)=x h_{\perp}^{(1)}(x, q)$. The first power correction introduces a transverse part to $A^{i a}$ so that these gluon distributions are no longer equal. The correction to $x G^{(1)}(x, q)$ and $x h_{\perp}^{(1)}(x, q)$ involves a correlator of four $A^{+}$in the proton. This is an independent function when color charge fluctuations are not Gaussian, and we have related it explicitly to overlap integrals of the LFWF of the proton. Numerically, we find that for $q \gtrsim 0.5 \mathrm{GeV}$ the higher twist correction is small and "polarization is close to maximal. Hence, a measurement of $x h_{\perp}^{(1)}(x, q)$ at an EIC appears promising.

Throughout the paper we have approximated the proton in terms of its valence quark Fock state. It will be important to include the $|q q q g\rangle$ Fock state, too, where the gluon is not necessarily soft. This may affect color charge correlations which probe high parton transverse momenta, and should improve the matching to small- $x$ BK evolution. Work in that direction is in progress.

\section{ACKNOWLEDGMENTS}

We thank Y. Hatta and L. Motyka for useful comments. Figures 1 and 9 were prepared with Jaxodraw [44]. A. D. acknowledges support by the DOE Office of Nuclear Physics through Grant No. DE-FG02-09ER41620 and from The City University of New York through the PSC-CUNY Research Grant No. 62098-00 50. V. S. acknowledges support by the DOE Office of Nuclear Physics through Grant No. DE-SC0020081. V.S. thanks the ExtreMe Matter Institute EMMI (GSI Helmholtzzentrum für Schwerionenforschung, Darmstadt, Germany) for partial support and hospitality. T.S. is supported by the Polish
National Science Center (NCN) Grants No. 2017/27/B/ST2/ 02755 and No. 2019/32/C/ST2/00202.

\section{APPENDIX A: SIMPLE MODEL WAVE FUNCTION}

For numerical estimates we employ the "harmonic oscillator" model wave function of Brodsky and Schlumpf [27],

$\psi_{\text {H.O. }}\left(x_{1}, \vec{k}_{1} ; x_{2}, \vec{k}_{2} ; x_{3}, \vec{k}_{3}\right)=N_{\text {H.O. }} \exp \left(-\mathcal{M}^{2} / 2 \beta^{2}\right)$.

The invariant mass $\mathcal{M}$ of the configuration is given by

$$
\mathcal{M}^{2}=\sum_{i=1}^{3} \frac{\vec{k}_{i}^{2}+m^{2}}{x_{i}} .
$$

$\beta$ determines the typical transverse momentum of quarks in the proton. The parameters $\beta$ and $m^{2}$ were determined in Ref. [27] as $m=0.26 \mathrm{GeV}, \beta=0.55 \mathrm{GeV}$. The normalization constant $N_{\text {H.O. }}$ is obtained from the normalization condition (2).

The above simple model wave function allows us to perform analytically parts of the evaluation of the correlators of + color currents in the proton; cf. Eqs. (B3), (B10), (B11), (B13). This simplifies the numerical computations significantly. Other models and parameter sets can be found in Refs. [45].

\section{APPENDIX B: COLOR CHARGE CORRELATORS}

Following Ref. [3] we introduce the color charge density operators corresponding to the light cone plus a component of the quark currents

$$
\rho^{a}\left(x_{k} \ll 1, \vec{k}\right)=g \sum_{i, j} \int \frac{\mathrm{d} x_{q}}{x_{q}} \int \frac{\mathrm{d}^{2} q}{16 \pi^{3}} b_{x_{q}, \vec{q}, i}^{\dagger} b_{x_{q}, \vec{k}+\vec{q}, j}\left(t^{a}\right)_{i j} .
$$

$b_{q, i}^{\dagger}$ and $b_{q, i}$ denote creation and annihilation operators for quarks with plus momentum $q^{+}=x_{q} P^{+}$, transverse momentum $\vec{q}$, and color $i$. Note that this neglects contributions from antiquarks and gluons which we assume are small at $x_{k} \sim 0.1$. We also neglect longitudinal momentum transfer to the quarks and use the kinematic approximation where $x_{k} \sim 0.1 \ll 1$. This allows us to simplify the color charge operators as indicated above. 
The expectation value of a single color charge operator in the proton is given by ${ }^{8}$

$$
\begin{aligned}
\left\langle\rho^{a}\left(-\vec{K}_{T}\right)\right\rangle= & g \operatorname{tr} t^{a} \int \mathrm{d} x_{1} \mathrm{~d} x_{2} \mathrm{~d} x_{3} \delta\left(1-x_{1}-x_{2}-x_{3}\right) \int \frac{\mathrm{d}^{2} p_{1} \mathrm{~d}^{2} p_{2} \mathrm{~d}^{2} p_{3}}{\left(16 \pi^{3}\right)^{2}} \delta\left(\vec{p}_{1}+\vec{p}_{2}+\vec{p}_{3}\right) \\
& \times \psi^{*}\left(\vec{p}_{1}+\left(1-x_{1}\right) \vec{K}_{T}, \vec{p}_{2}-x_{2} \vec{K}_{T}, \vec{p}_{3}-x_{3} \vec{K}_{T}\right) \psi\left(\vec{p}_{1}, \vec{p}_{2}, \vec{p}_{3}\right) \\
= & g \operatorname{tr} t^{a} \int \mathrm{d} x_{1} \int \frac{\mathrm{d}^{2} p_{1}}{(2 \pi)^{2}} W_{K_{T}}^{(1)}\left(x_{1}, \vec{p}_{1}+\vec{K}_{T}\right) .
\end{aligned}
$$

For brevity we omit the momentum fractions $x_{1}, x_{2}, x_{3}$ from the list of arguments of $\psi$ and $\psi^{*}$ since we employ the eikonal approximation. Here, $W_{K_{T}}^{(1)}\left(x_{1}, \vec{p}_{1}+\vec{K}_{T}\right)$ is the one-body quark GTMD/Wigner distribution for momentum transfer $K_{T}$; one may Fourier transform it from $\vec{K}_{T}$-space to $\vec{b}$-space. Of course, $\left\langle\rho^{a}\left(-\vec{K}_{T}\right)\right\rangle$ vanishes due to color neutrality.

The correlator of two color charge density operators is given by [3]

$$
\begin{aligned}
\left\langle\rho^{a}\left(\vec{q}_{1}\right) \rho^{b}\left(\vec{q}_{2}\right)\right\rangle= & g^{2} \operatorname{tr} t^{a} t^{b} \int \mathrm{d} x_{1} \mathrm{~d} x_{2} \mathrm{~d} x_{3} \delta\left(1-x_{1}-x_{2}-x_{3}\right) \int \frac{\mathrm{d}^{2} p_{1} \mathrm{~d}^{2} p_{2} \mathrm{~d}^{2} p_{3}}{\left(16 \pi^{3}\right)^{2}} \delta\left(\vec{p}_{1}+\vec{p}_{2}+\vec{p}_{3}\right) \\
& \times\left[\psi^{*}\left(\vec{p}_{1}-\vec{q}_{1}-\vec{q}_{2}-x_{1} \vec{K}_{T}, \vec{p}_{2}-x_{2} \vec{K}_{T}, \vec{p}_{3}-x_{3} \vec{K}_{T}\right)\right. \\
& \left.-\psi^{*}\left(\vec{p}_{1}-\vec{q}_{1}-x_{1} \vec{K}_{T}, \vec{p}_{2}-\vec{q}_{2}-x_{2} \vec{K}_{T}, \vec{p}_{3}-x_{3} \vec{K}_{T}\right)\right] \psi\left(\vec{p}_{1}, \vec{p}_{2}, \vec{p}_{3}\right) \\
\equiv & \frac{1}{2} \delta^{a b} g^{2} G_{2}\left(\vec{q}_{1}, \vec{q}_{2}\right) .
\end{aligned}
$$

$\vec{K}_{T}$ is the total momentum transfer to the proton; by conservation of transverse momentum we have that $\vec{K}_{T}=-\left(\vec{q}_{1}+\vec{q}_{2}\right)$. Similarly, in all charge correlators below $\vec{K}_{T}+\sum_{i} \vec{q}_{i}=0$. Up to a conventional factor of $(-i)^{2}$ which we write explicitly in the exponent of the Wilson lines (6), this result coincides with the two-gluon exchange proton impact factor given in Refs. [46,47].

In the limit where all $q_{i}$ far exceed the typical transverse momentum of quarks in the proton, while $K_{T} \ll q_{i}$, this correlator, as well as higher correlators introduced below, approach a universal limit given by a one-body GPD:

$$
\begin{aligned}
G_{2}\left(\vec{q}_{1}, \vec{q}_{2}\right) \rightarrow & \int \mathrm{d} x_{1} \mathrm{~d} x_{2} \mathrm{~d} x_{3} \delta\left(1-x_{1}-x_{2}-x_{3}\right) \int \frac{\mathrm{d}^{2} p_{1} \mathrm{~d}^{2} p_{2} \mathrm{~d}^{2} p_{3}}{\left(16 \pi^{3}\right)^{2}} \delta\left(\vec{p}_{1}+\vec{p}_{2}+\vec{p}_{3}\right) \\
& \times \psi^{*}\left(\vec{p}_{1}-\vec{q}_{1}-\vec{q}_{2}-x_{1} \vec{K}_{T}, \vec{p}_{2}-x_{2} \vec{K}_{T}, \vec{p}_{3}-x_{3} \vec{K}_{T}\right) \psi\left(\vec{p}_{1}, \vec{p}_{2}, \vec{p}_{3}\right) \\
= & \int \mathrm{d} x_{1} \int \frac{\mathrm{d}^{2} p_{1}}{(2 \pi)^{2}} W_{K_{T}}^{(1)}\left(x_{1}, \vec{p}_{1}+\vec{K}_{T}\right), \quad\left(K_{T} \ll q_{1}, q_{2}\right) .
\end{aligned}
$$

The term "one-body GPD" refers to the fact that both color charge operators act on one and the same quark and one may integrate out the spectator quarks. On the other hand, when the probes share a large momentum transfer $\vec{K}_{T}$ the dominant contribution is due to the diagram where the two gluons attach to different quarks in the proton, i.e., to the two-body representation of $\rho^{a}\left(\vec{q}_{1}\right) \rho^{b}\left(\vec{q}_{2}\right)$ which gives the second term in Eq. (B3) [29]:

$$
\begin{aligned}
G_{2}\left(\vec{q}_{1}, \vec{q}_{2}\right) \rightarrow & -\int \mathrm{d} x_{1} \mathrm{~d} x_{2} \mathrm{~d} x_{3} \delta\left(1-x_{1}-x_{2}-x_{3}\right) \int \frac{\mathrm{d}^{2} p_{1} \mathrm{~d}^{2} p_{2} \mathrm{~d}^{2} p_{3}}{\left(16 \pi^{3}\right)^{2}} \delta\left(\vec{p}_{1}+\vec{p}_{2}+\vec{p}_{3}\right) \\
& \times \psi^{*}\left(\vec{p}_{1}-\vec{q}_{1}-x_{1} \vec{K}_{T}, \vec{p}_{2}-\vec{q}_{2}-x_{2} \vec{K}_{T}, \vec{p}_{3}-x_{3} \vec{K}_{T}\right) \psi\left(\vec{p}_{1}, \vec{p}_{2}, \vec{p}_{3}\right) \\
= & \int \mathrm{d} x_{1} \int \frac{\mathrm{d}^{2} p_{1}}{(2 \pi)^{2}} \int \mathrm{d} x_{2} \int \frac{\mathrm{d}^{2} p_{2}}{(2 \pi)^{2}} W_{K_{T}}^{(2)}\left(x_{1}, \vec{p}_{1}-\vec{q}_{1}, x_{2}, \vec{p}_{2}-\vec{q}_{2}\right) \quad\left(\vec{q}_{1}, \vec{q}_{2} \sim-\vec{K}_{T} / 2\right) .
\end{aligned}
$$

\footnotetext{
${ }^{8}\langle\cdots\rangle$ corresponds to $\langle K|\cdots| P\rangle$ stripped of the $\delta$-functions expressing conservation of transverse and plus momentum, e.g., $\left\langle K\left|\rho^{a}(\vec{q})\right| P\right\rangle=16 \pi^{3} P^{+} \delta\left(P^{+}-K^{+}\right) \delta\left(\vec{K}_{T}+\vec{q}\right)\left\langle\rho^{a}(\vec{q})\right\rangle$, where we set $\vec{P}_{T}=0$ for the incoming proton.
} 
This involves a two-body GTMD or Wigner distribution. The $n$-body diagrams are important for exclusive photoproduction of charmonium at large $-t$ [29].

We now proceed with cubic and quartic color charge correlators. The fact that $\left\langle\rho^{a}\left(\vec{q}_{1}\right) \rho^{b}\left(\vec{q}_{2}\right) \rho^{c}\left(\vec{q}_{3}\right)\right\rangle$ is not zero shows that color charge fluctuations are not Gaussian. The $C$-odd part of the cubic correlator is given by [3]

$$
\begin{aligned}
\left\langle\rho^{a}\left(\vec{q}_{1}\right) \rho^{b}\left(\vec{q}_{2}\right) \rho^{c}\left(\vec{q}_{3}\right)\right\rangle_{C=-}= & \frac{1}{4} d^{a b c} g^{3} \int \mathrm{d} x_{1} \mathrm{~d} x_{2} \mathrm{~d} x_{3} \delta\left(1-x_{1}-x_{2}-x_{3}\right) \int \frac{\mathrm{d}^{2} p_{1} \mathrm{~d}^{2} p_{2} \mathrm{~d}^{2} p_{3}}{\left(16 \pi^{3}\right)^{2}} \delta\left(\vec{p}_{1}+\vec{p}_{2}+\vec{p}_{3}\right) \\
& \times\left[\psi^{*}\left(\vec{p}_{1}-\vec{q}_{1}-\vec{q}_{2}-\vec{q}_{3}-x_{1} \vec{K}_{\perp}, \vec{p}_{2}-x_{2} \vec{K}_{\perp}, \vec{p}_{3}-x_{3} \vec{K}_{\perp}\right)\right. \\
& -\psi^{*}\left(\vec{p}_{1}-\vec{q}_{1}-x_{1} \vec{K}_{\perp}, \vec{p}_{2}-\vec{q}_{2}-\vec{q}_{3}-x_{2} \vec{K}_{\perp}, \vec{p}_{3}-x_{3} \vec{K}_{\perp}\right) \\
& -\psi^{*}\left(\vec{p}_{1}-\vec{q}_{1}-\vec{q}_{3}-x_{1} \vec{K}_{\perp}, \vec{p}_{2}-\vec{q}_{2}-x_{2} \vec{K}_{\perp}, \vec{p}_{3}-x_{3} \vec{K}_{\perp}\right) \\
& -\psi^{*}\left(\vec{p}_{1}-\vec{q}_{1}-\vec{q}_{2}-x_{1} \vec{K}_{\perp}, \vec{p}_{2}-\vec{q}_{3}-x_{2} \vec{K}_{\perp}, \vec{p}_{3}-x_{3} \vec{K}_{\perp}\right) \\
& \left.+2 \psi^{*}\left(\vec{p}_{1}-\vec{q}_{1}-x_{1} \vec{K}_{\perp}, \vec{p}_{2}-\vec{q}_{2}-x_{2} \vec{K}_{\perp}, \vec{p}_{3}-\vec{q}_{3}-x_{3} \vec{K}_{\perp}\right)\right] \psi\left(\vec{p}_{1}, \vec{p}_{2}, \vec{p}_{3}\right) \\
\equiv & \frac{1}{4} d^{a b c} g^{3} G_{3}^{-}\left(\vec{q}_{1}, \vec{q}_{2}, \vec{q}_{3}\right) .
\end{aligned}
$$

Again, this expression agrees with the $C$-odd three-gluon exchange proton impact factor $E_{3 ; 0}$ by Bartels and Motyka [46] (also see Refs. [48]) up to a conventional factor of $(-i)^{3}$.

$G_{3}^{-}$can be expressed in terms of two-gluon exchange correlators $G_{2}$, where two of the three gluons are "paired up," plus a genuine three-body contribution which enforces the Ward identity (vanishing of $G_{3}^{-}$) when either one $\vec{q}_{i} \rightarrow 0$ :

$$
\begin{aligned}
G_{3}^{-}\left(\vec{q}_{1}, \vec{q}_{2}, \vec{q}_{3}\right)= & G_{2}\left(\vec{q}_{1}+\vec{q}_{2}, \vec{q}_{3}\right)+G_{2}\left(\vec{q}_{1}+\vec{q}_{3}, \vec{q}_{2}\right)+G_{2}\left(\vec{q}_{2}+\vec{q}_{3}, \vec{q}_{1}\right) \\
& -2 \int \mathrm{d} x_{1} \mathrm{~d} x_{2} \mathrm{~d} x_{3} \delta\left(1-x_{1}-x_{2}-x_{3}\right) \int \frac{\mathrm{d}^{2} p_{1} \mathrm{~d}^{2} p_{2} \mathrm{~d}^{2} p_{3}}{\left(16 \pi^{3}\right)^{2}} \delta\left(\vec{p}_{1}+\vec{p}_{2}+\vec{p}_{3}\right) \\
& \times\left[\psi^{*}\left(\vec{p}_{1}-\vec{q}_{1}-\vec{q}_{2}-\vec{q}_{3}-x_{1} \vec{K}_{\perp}, \vec{p}_{2}-x_{2} \vec{K}_{\perp}, \vec{p}_{3}-x_{3} \vec{K}_{\perp}\right)\right. \\
& \left.-\psi^{*}\left(\vec{p}_{1}-\vec{q}_{1}-x_{1} \vec{K}_{\perp}, \vec{p}_{2}-\vec{q}_{2}-x_{2} \vec{K}_{\perp}, \vec{p}_{3}-\vec{q}_{3}-x_{3} \vec{K}_{\perp}\right)\right] \psi\left(\vec{p}_{1}, \vec{p}_{2}, \vec{p}_{3}\right) .
\end{aligned}
$$

For completeness we also give the $C$-even (or negative signature) part of the cubic correlator although it is not needed for the dipole scattering amplitude:

$$
\begin{aligned}
\left\langle\rho^{a}\left(\vec{q}_{1}\right) \rho^{b}\left(\vec{q}_{2}\right) \rho^{c}\left(\vec{q}_{3}\right)\right\rangle_{C=+}= & \frac{i}{4} f^{a b c} g^{3} \int \mathrm{d} x_{1} \mathrm{~d} x_{2} \mathrm{~d} x_{3} \delta\left(1-x_{1}-x_{2}-x_{3}\right) \int \frac{\mathrm{d}^{2} p_{1} \mathrm{~d}^{2} p_{2} \mathrm{~d}^{2} p_{3}}{\left(16 \pi^{3}\right)^{2}} \delta\left(\vec{p}_{1}+\vec{p}_{2}+\vec{p}_{3}\right) \\
& \times\left[\psi^{*}\left(\vec{p}_{1}-\vec{q}_{1}-\vec{q}_{2}-\vec{q}_{3}-x_{1} \vec{K}_{\perp}, \vec{p}_{2}-x_{2} \vec{K}_{\perp}, \vec{p}_{3}-x_{3} \vec{K}_{\perp}\right)\right. \\
& -\psi^{*}\left(\vec{p}_{1}-\vec{q}_{2}-\vec{q}_{3}-x_{1} \vec{K}_{\perp}, \vec{p}_{2}-\vec{q}_{1}-x_{2} \vec{K}_{\perp}, \vec{p}_{3}-x_{3} \vec{K}_{\perp}\right) \\
& +\psi^{*}\left(\vec{p}_{1}-\vec{q}_{1}-\vec{q}_{3}-x_{1} \vec{K}_{\perp}, \vec{p}_{2}-\vec{q}_{2}-x_{2} \vec{K}_{\perp}, \vec{p}_{3}-x_{3} \vec{K}_{\perp}\right) \\
& \left.-\psi^{*}\left(\vec{p}_{1}-\vec{q}_{1}-\vec{q}_{2}-x_{1} \vec{K}_{\perp}, \vec{p}_{2}-\vec{q}_{3}-x_{2} \vec{K}_{\perp}, \vec{p}_{3}-x_{3} \vec{K}_{\perp}\right)\right] \psi\left(\vec{p}_{1}, \vec{p}_{2}, \vec{p}_{3}\right) \\
\equiv & \frac{i}{4} f^{a b c} g^{3} G_{3}^{+}\left(\vec{q}_{1}, \vec{q}_{2}, \vec{q}_{3}\right) .
\end{aligned}
$$

$G_{3}^{+}$can be fully decomposed into twp-gluon exchanges, similar to Reggeized gluon exchanges at small- $x[26,46]$ :

$$
G_{3}^{+}\left(\vec{q}_{1}, \vec{q}_{2}, \vec{q}_{3}\right)=G_{2}\left(\vec{q}_{1}+\vec{q}_{2}, \vec{q}_{3}\right)-G_{2}\left(\vec{q}_{1}+\vec{q}_{3}, \vec{q}_{2}\right)+G_{2}\left(\vec{q}_{1}, \vec{q}_{2}+\vec{q}_{3}\right)
$$

This vanishes when the transverse momentum of the first or last gluon $\left(\vec{q}_{1}\right.$ resp. $\left.\vec{q}_{3}\right)$ is taken to zero but not for $\vec{q}_{2} \rightarrow 0$ [26]. 
Lastly, the correlator of four color charge operators is given by

$$
\begin{aligned}
& \left\langle\rho^{a}\left(\vec{q}_{1}\right) \rho^{b}\left(\vec{q}_{2}\right) \rho^{c}\left(\vec{q}_{3}\right) \rho^{d}\left(\vec{q}_{4}\right)\right\rangle \\
& =g^{4} \int \mathrm{d} x_{1} \mathrm{~d} x_{2} \mathrm{~d} x_{3} \delta\left(1-x_{1}-x_{2}-x_{3}\right) \int \frac{\mathrm{d}^{2} p_{1} \mathrm{~d}^{2} p_{2} \mathrm{~d}^{2} p_{3}}{\left(16 \pi^{3}\right)^{2}} \delta\left(\vec{p}_{1}+\vec{p}_{2}+\vec{p}_{3}\right) \psi\left(\vec{p}_{1}, \vec{p}_{2}, \vec{p}_{3}\right) \\
& \times\left\{\operatorname{tr} t^{a} t^{b} t^{c} t^{d} \psi^{*}\left(\vec{p}_{1}-\vec{q}_{1}-\vec{q}_{2}-\vec{q}_{3}-\vec{q}_{4}-x_{1} \vec{K}_{T}, \vec{p}_{2}-x_{2} \vec{K}_{T}, \vec{p}_{3}-x_{3} \vec{K}_{T}\right)\right. \\
& +\left(\operatorname{tr} t^{a} t^{b} \operatorname{tr} t^{c} t^{d}-\operatorname{tr} t^{a} t^{b} t^{c} t^{d}\right) \psi^{*}\left(\vec{p}_{1}-\vec{q}_{1}-\vec{q}_{2}-x_{1} \vec{K}_{T}, \vec{p}_{2}-\vec{q}_{3}-\vec{q}_{4}-x_{2} \vec{K}_{T}, \vec{p}_{3}-x_{3} \vec{K}_{T}\right) \\
& +\left(\operatorname{tr} t^{a} t^{c} \operatorname{tr} t^{b} t^{d}-\operatorname{tr} t^{a} t^{c} t^{b} t^{d}\right) \psi^{*}\left(\vec{p}_{1}-\vec{q}_{1}-\vec{q}_{3}-x_{1} \vec{K}_{T}, \vec{p}_{2}-\vec{q}_{2}-\vec{q}_{4}-x_{2} \vec{K}_{T}, \vec{p}_{3}-x_{3} \vec{K}_{T}\right) \\
& +\left(\operatorname{tr} t^{a} t^{d} \operatorname{tr} t^{b} t^{c}-\operatorname{tr} t^{a} t^{d} t^{b} t^{c}\right) \psi^{*}\left(\vec{p}_{1}-\vec{q}_{1}-\vec{q}_{4}-x_{1} \vec{K}_{T}, \vec{p}_{2}-\vec{q}_{2}-\vec{q}_{3}-x_{2} \vec{K}_{T}, \vec{p}_{3}-x_{3} \vec{K}_{T}\right) \\
& -\operatorname{tr} t^{a} t^{b} t^{c} t^{d} \psi^{*}\left(\vec{p}_{1}-\vec{q}_{1}-\vec{q}_{2}-\vec{q}_{3}-x_{1} \vec{K}_{T}, \vec{p}_{2}-\vec{q}_{4}-x_{2} \vec{K}_{T}, \vec{p}_{3}-x_{3} \vec{K}_{T}\right) \\
& -\operatorname{tr} t^{a} t^{b} t^{c} t^{d} \psi^{*}\left(\vec{p}_{1}-\vec{q}_{1}-x_{1} \vec{K}_{T}, \vec{p}_{2}-\vec{q}_{2}-\vec{q}_{3}-\vec{q}_{4}-x_{2} \vec{K}_{T}, \vec{p}_{3}-x_{3} \vec{K}_{T}\right) \\
& -\operatorname{tr} t^{a} t^{b} t^{d} t^{c} \psi^{*}\left(\vec{p}_{1}-\vec{q}_{1}-\vec{q}_{2}-\vec{q}_{4}-x_{1} \vec{K}_{T}, \vec{p}_{2}-\vec{q}_{3}-x_{2} \vec{K}_{T}, \vec{p}_{3}-x_{3} \vec{K}_{T}\right) \\
& -\operatorname{tr} t^{a} t^{c} t^{d} t^{b} \psi^{*}\left(\vec{p}_{1}-\vec{q}_{1}-\vec{q}_{3}-\vec{q}_{4}-x_{1} \vec{K}_{T}, \vec{p}_{2}-\vec{q}_{2}-x_{2} \vec{K}_{T}, \vec{p}_{3}-x_{3} \vec{K}_{T}\right) \\
& +\left(\operatorname{tr} t^{a} t^{b} t^{c} t^{d}+\operatorname{tr} t^{a} t^{b} t^{d} t^{c}-\operatorname{tr} t^{a} t^{b} \operatorname{tr} t^{c} t^{d}\right) \psi^{*}\left(\vec{p}_{1}-\vec{q}_{1}-\vec{q}_{2}-x_{1} \vec{K}_{T}, \vec{p}_{2}-\vec{q}_{3}-x_{2} \vec{K}_{T}, \vec{p}_{3}-\vec{q}_{4}-x_{3} \vec{K}_{T}\right) \\
& +\left(\operatorname{tr} t^{a} t^{c} t^{b} t^{d}+\operatorname{tr} t^{a} t^{c} t^{d} t^{b}-\operatorname{tr} t^{a} t^{c} \operatorname{tr} t^{b} t^{d}\right) \psi^{*}\left(\vec{p}_{1}-\vec{q}_{1}-\vec{q}_{3}-x_{1} \vec{K}_{T}, \vec{p}_{2}-\vec{q}_{2}-x_{2} \vec{K}_{T}, \vec{p}_{3}-\vec{q}_{4}-x_{3} \vec{K}_{T}\right) \\
& +\left(\operatorname{tr} t^{a} t^{d} t^{b} t^{c}+\operatorname{tr} t^{a} t^{d} t^{c} t^{b}-\operatorname{tr} t^{a} t^{d} \operatorname{tr} t^{b} t^{c}\right) \psi^{*}\left(\vec{p}_{1}-\vec{q}_{1}-\vec{q}_{4}-x_{1} \vec{K}_{T}, \vec{p}_{2}-\vec{q}_{2}-x_{2} \vec{K}_{T}, \vec{p}_{3}-\vec{q}_{3}-x_{3} \vec{K}_{T}\right) \\
& +\left(\operatorname{tr} t^{a} t^{b} t^{c} t^{d}+\operatorname{tr} t^{a} t^{d} t^{b} t^{c}-\operatorname{tr} t^{a} t^{d} \operatorname{tr} t^{b} t^{c}\right) \psi^{*}\left(\vec{p}_{1}-\vec{q}_{1}-x_{1} \vec{K}_{T}, \vec{p}_{2}-\vec{q}_{2}-\vec{q}_{3}-x_{2} \vec{K}_{T}, \vec{p}_{3}-\vec{q}_{4}-x_{3} \vec{K}_{T}\right) \\
& +\left(\operatorname{tr} t^{a} t^{b} t^{c} t^{d}+\operatorname{tr} t^{a} t^{c} t^{d} t^{b}-\operatorname{tr} t^{a} t^{b} \operatorname{tr} t^{c} t^{d}\right) \psi^{*}\left(\vec{p}_{1}-\vec{q}_{1}-x_{1} \vec{K}_{T}, \vec{p}_{2}-\vec{q}_{2}-x_{2} \vec{K}_{T}, \vec{p}_{3}-\vec{q}_{3}-\vec{q}_{4}-x_{3} \vec{K}_{T}\right) \\
& \left.+\left(\operatorname{tr} t^{a} t^{b} t^{d} t^{c}+\operatorname{tr} t^{a} t^{c} t^{b} t^{d}-\operatorname{tr} t^{a} t^{c} \operatorname{tr} t^{b} t^{d}\right) \psi^{*}\left(\vec{p}_{1}-\vec{q}_{1}-x_{1} \vec{K}_{T}, \vec{p}_{2}-\vec{q}_{2}-\vec{q}_{4}-x_{2} \vec{K}_{T}, \vec{p}_{3}-\vec{q}_{3}-x_{3} \vec{K}_{T}\right)\right\},
\end{aligned}
$$

where $\vec{K}_{T} \equiv-\left(\vec{q}_{1}+\vec{q}_{2}+\vec{q}_{3}+\vec{q}_{4}\right)$. Note that it is not equal to a sum over all permutations of pairwise contractions, confirming that color charge fluctuations are not Gaussian.

We can decompose this correlator into $C$-even and odd parts. Charge conjugation transforms $t^{a} \rightarrow-t^{a T}$ so that $\operatorname{tr} t^{a} t^{b} t^{c} t^{d} \rightarrow \operatorname{tr} t^{d} t^{c} t^{b} t^{a}=\operatorname{tr} t^{b} t^{a} t^{d} t^{c}$ which corresponds to the permutations $a \leftrightarrow b, c \leftrightarrow d$. Hence, using $\operatorname{tr} t^{a} t^{b} t^{c} t^{d}=$ $(1 / 12) \delta^{a b} \delta^{c d}+(1 / 8)\left(d^{a b e}+i f^{a b e}\right)\left(d^{c d e}+i f^{c d e}\right)$ we see that the $C$-even pieces of $\operatorname{tr} t^{a} t^{b} t^{c} t^{d}$ correspond to the color structures $\delta^{a b} \delta^{c d}, d^{a b e} d^{c d e}$, and $f^{a b e} f^{c d e}$, while the $C$-odd pieces correspond to $i d^{a b e} f^{c d e}$.

Therefore, the $C$-even parts of $\left\langle\rho^{4}\right\rangle$ are

$$
\begin{aligned}
\left\langle\rho^{a}\left(\vec{q}_{1}\right) \rho^{b}\left(\vec{q}_{2}\right) \rho^{c}\left(\vec{q}_{3}\right) \rho^{d}\left(\vec{q}_{4}\right)\right\rangle_{f f}= & -\frac{1}{8} g^{4} \int \mathrm{d} x_{1} \mathrm{~d} x_{2} \mathrm{~d} x_{3} \delta\left(1-x_{1}-x_{2}-x_{3}\right) \int \frac{\mathrm{d}^{2} p_{1} \mathrm{~d}^{2} p_{2} \mathrm{~d}^{2} p_{3}}{\left(16 \pi^{3}\right)^{2}} \delta\left(\vec{p}_{1}+\vec{p}_{2}+\vec{p}_{3}\right) \psi\left(\vec{p}_{1}, \vec{p}_{2}, \vec{p}_{3}\right) \\
& \times\left\{f^{a b e} f^{c d e} \psi^{*}\left(\vec{p}_{1}-\vec{q}_{1}-\vec{q}_{2}-\vec{q}_{3}-\vec{q}_{4}-x_{1} \vec{K}_{T}, \vec{p}_{2}-x_{2} \vec{K}_{T}, \vec{p}_{3}-x_{3} \vec{K}_{T}\right)\right. \\
& -f^{a b e} f^{c d e} \psi^{*}\left(\vec{p}_{1}-\vec{q}_{1}-\vec{q}_{2}-x_{1} \vec{K}_{T}, \vec{p}_{2}-\vec{q}_{3}-\vec{q}_{4}-x_{2} \vec{K}_{T}, \vec{p}_{3}-x_{3} \vec{K}_{T}\right) \\
& -f^{a c e} f^{b d e} \psi^{*}\left(\vec{p}_{1}-\vec{q}_{1}-\vec{q}_{3}-x_{1} \vec{K}_{T}, \vec{p}_{2}-\vec{q}_{2}-\vec{q}_{4}-x_{2} \vec{K}_{T}, \vec{p}_{3}-x_{3} \vec{K}_{T}\right) \\
& -f^{a d e} f^{b c e} \psi^{*}\left(\vec{p}_{1}-\vec{q}_{1}-\vec{q}_{4}-x_{1} \vec{K}_{T}, \vec{p}_{2}-\vec{q}_{2}-\vec{q}_{3}-x_{2} \vec{K}_{T}, \vec{p}_{3}-x_{3} \vec{K}_{T}\right) \\
& -f^{a b e} f^{c d e} \psi^{*}\left(\vec{p}_{1}-\vec{q}_{1}-\vec{q}_{2}-\vec{q}_{3}-x_{1} \vec{K}_{T}, \vec{p}_{2}-\vec{q}_{4}-x_{2} \vec{K}_{T}, \vec{p}_{3}-x_{3} \vec{K}_{T}\right) \\
& -f^{a b e} f^{c d e} \psi^{*}\left(\vec{p}_{1}-\vec{q}_{1}-x_{1} \vec{K}_{T}, \vec{p}_{2}-\vec{q}_{2}-\vec{q}_{3}-\vec{q}_{4}-x_{2} \vec{K}_{T}, \vec{p}_{3}-x_{3} \vec{K}_{T}\right) \\
& -f^{a b e} f^{d c e} \psi^{*}\left(\vec{p}_{1}-\vec{q}_{1}-\vec{q}_{2}-\vec{q}_{4}-x_{1} \vec{K}_{T}, \vec{p}_{2}-\vec{q}_{3}-x_{2} \vec{K}_{T}, \vec{p}_{3}-x_{3} \vec{K}_{T}\right) \\
& \left.-f^{a c e} f^{d b e} \psi^{*}\left(\vec{p}_{1}-\vec{q}_{1}-\vec{q}_{3}-\vec{q}_{4}-x_{1} \vec{K}_{T}, \vec{p}_{2}-\vec{q}_{2}-x_{2} \vec{K}_{T}, \vec{p}_{3}-x_{3} \vec{K}_{T}\right)\right\},
\end{aligned}
$$


$\left\langle\rho^{a}\left(\vec{q}_{1}\right) \rho^{b}\left(\vec{q}_{2}\right) \rho^{c}\left(\vec{q}_{3}\right) \rho^{d}\left(\vec{q}_{4}\right)\right\rangle_{d d}=\frac{1}{8} g^{4} \int \mathrm{d} x_{1} \mathrm{~d} x_{2} \mathrm{~d} x_{3} \delta\left(1-x_{1}-x_{2}-x_{3}\right) \int \frac{\mathrm{d}^{2} p_{1} \mathrm{~d}^{2} p_{2} \mathrm{~d}^{2} p_{3}}{\left(16 \pi^{3}\right)^{2}} \delta\left(\vec{p}_{1}+\vec{p}_{2}+\vec{p}_{3}\right) \psi\left(\vec{p}_{1}, \vec{p}_{2}, \vec{p}_{3}\right)$

$$
\begin{aligned}
& \times\left\{d^{\text {abe }} d^{\text {cde }} \psi^{*}\left(\vec{p}_{1}-\vec{q}_{1}-\vec{q}_{2}-\vec{q}_{3}-\vec{q}_{4}-x_{1} \vec{K}_{T}, \vec{p}_{2}-x_{2} \vec{K}_{T}, \vec{p}_{3}-x_{3} \vec{K}_{T}\right)\right. \\
& -d^{\text {abe }} d^{c d e} \psi^{*}\left(\vec{p}_{1}-\vec{q}_{1}-\vec{q}_{2}-x_{1} \vec{K}_{T}, \vec{p}_{2}-\vec{q}_{3}-\vec{q}_{4}-x_{2} \vec{K}_{T}, \vec{p}_{3}-x_{3} \vec{K}_{T}\right) \\
& -d^{\text {ace }} d^{\text {bde }} \psi^{*}\left(\vec{p}_{1}-\vec{q}_{1}-\vec{q}_{3}-x_{1} \vec{K}_{T}, \vec{p}_{2}-\vec{q}_{2}-\vec{q}_{4}-x_{2} \vec{K}_{T}, \vec{p}_{3}-x_{3} \vec{K}_{T}\right) \\
& -d^{\text {ade }} d^{b c e} \psi^{*}\left(\vec{p}_{1}-\vec{q}_{1}-\vec{q}_{4}-x_{1} \vec{K}_{T}, \vec{p}_{2}-\vec{q}_{2}-\vec{q}_{3}-x_{2} \vec{K}_{T}, \vec{p}_{3}-x_{3} \vec{K}_{T}\right) \\
& -d^{\text {abe }} d^{c d e} \psi^{*}\left(\vec{p}_{1}-\vec{q}_{1}-\vec{q}_{2}-\vec{q}_{3}-x_{1} \vec{K}_{T}, \vec{p}_{2}-\vec{q}_{4}-x_{2} \vec{K}_{T}, \vec{p}_{3}-x_{3} \vec{K}_{T}\right) \\
& -d^{\text {abe }} d^{c d e} \psi^{*}\left(\vec{p}_{1}-\vec{q}_{1}-x_{1} \vec{K}_{T}, \vec{p}_{2}-\vec{q}_{2}-\vec{q}_{3}-\vec{q}_{4}-x_{2} \vec{K}_{T}, \vec{p}_{3}-x_{3} \vec{K}_{T}\right) \\
& -d^{\text {abe }} d^{d c e} \psi^{*}\left(\vec{p}_{1}-\vec{q}_{1}-\vec{q}_{2}-\vec{q}_{4}-x_{1} \vec{K}_{T}, \vec{p}_{2}-\vec{q}_{3}-x_{2} \vec{K}_{T}, \vec{p}_{3}-x_{3} \vec{K}_{T}\right) \\
& -d^{\text {ace }} d^{\text {bde }} \psi^{*}\left(\vec{p}_{1}-\vec{q}_{1}-\vec{q}_{3}-\vec{q}_{4}-x_{1} \vec{K}_{T}, \vec{p}_{2}-\vec{q}_{2}-x_{2} \vec{K}_{T}, \vec{p}_{3}-x_{3} \vec{K}_{T}\right) \\
& +2 d^{\text {abe }} d^{c d e} \psi^{*}\left(\vec{p}_{1}-\vec{q}_{1}-\vec{q}_{2}-x_{1} \vec{K}_{T}, \vec{p}_{2}-\vec{q}_{3}-x_{2} \vec{K}_{T}, \vec{p}_{3}-\vec{q}_{4}-x_{3} \vec{K}_{T}\right) \\
& +2 d^{\text {ace }} d^{\text {bde }} \psi^{*}\left(\vec{p}_{1}-\vec{q}_{1}-\vec{q}_{3}-x_{1} \vec{K}_{T}, \vec{p}_{2}-\vec{q}_{2}-x_{2} \vec{K}_{T}, \vec{p}_{3}-\vec{q}_{4}-x_{3} \vec{K}_{T}\right) \\
& +2 d^{\text {ade }} d^{\text {bce }} \psi^{*}\left(\vec{p}_{1}-\vec{q}_{1}-\vec{q}_{4}-x_{1} \vec{K}_{T}, \vec{p}_{2}-\vec{q}_{2}-x_{2} \vec{K}_{T}, \vec{p}_{3}-\vec{q}_{3}-x_{3} \vec{K}_{T}\right) \\
& +2 d^{\text {bce }} d^{\text {ade }} \psi^{*}\left(\vec{p}_{1}-\vec{q}_{1}-x_{1} \vec{K}_{T}, \vec{p}_{2}-\vec{q}_{2}-\vec{q}_{3}-x_{2} \vec{K}_{T}, \vec{p}_{3}-\vec{q}_{4}-x_{3} \vec{K}_{T}\right) \\
& +2 d^{\text {abe }} d^{\text {cde }} \psi^{*}\left(\vec{p}_{1}-\vec{q}_{1}-x_{1} \vec{K}_{T}, \vec{p}_{2}-\vec{q}_{2}-x_{2} \vec{K}_{T}, \vec{p}_{3}-\vec{q}_{3}-\vec{q}_{4}-x_{3} \vec{K}_{T}\right) \\
& \left.+2 d^{\text {ace }} d^{\text {bde }} \psi^{*}\left(\vec{p}_{1}-\vec{q}_{1}-x_{1} \vec{K}_{T}, \vec{p}_{2}-\vec{q}_{2}-\vec{q}_{4}-x_{2} \vec{K}_{T}, \vec{p}_{3}-\vec{q}_{3}-x_{3} \vec{K}_{T}\right)\right\},
\end{aligned}
$$

and

$\left\langle\rho^{a}\left(\vec{q}_{1}\right) \rho^{b}\left(\vec{q}_{2}\right) \rho^{c}\left(\vec{q}_{3}\right) \rho^{d}\left(\vec{q}_{4}\right)\right\rangle_{\delta \delta}=\frac{1}{12} g^{4} \int \mathrm{d} x_{1} \mathrm{~d} x_{2} \mathrm{~d} x_{3} \delta\left(1-x_{1}-x_{2}-x_{3}\right) \int \frac{\mathrm{d}^{2} p_{1} \mathrm{~d}^{2} p_{2} \mathrm{~d}^{2} p_{3}}{\left(16 \pi^{3}\right)^{2}} \delta\left(\vec{p}_{1}+\vec{p}_{2}+\vec{p}_{3}\right) \psi\left(\vec{p}_{1}, \vec{p}_{2}, \vec{p}_{3}\right)$

$$
\begin{aligned}
& \times\left\{\delta^{a b} \delta^{c d} \psi^{*}\left(\vec{p}_{1}-\vec{q}_{1}-\vec{q}_{2}-\vec{q}_{3}-\vec{q}_{4}-x_{1} \vec{K}_{T}, \vec{p}_{2}-x_{2} \vec{K}_{T}, \vec{p}_{3}-x_{3} \vec{K}_{T}\right)\right. \\
& +2 \delta^{a b} \delta^{c d} \psi^{*}\left(\vec{p}_{1}-\vec{q}_{1}-\vec{q}_{2}-x_{1} \vec{K}_{T}, \vec{p}_{2}-\vec{q}_{3}-\vec{q}_{4}-x_{2} \vec{K}_{T}, \vec{p}_{3}-x_{3} \vec{K}_{T}\right) \\
& +2 \delta^{a c} \delta^{b d} \psi^{*}\left(\vec{p}_{1}-\vec{q}_{1}-\vec{q}_{3}-x_{1} \vec{K}_{T}, \vec{p}_{2}-\vec{q}_{2}-\vec{q}_{4}-x_{2} \vec{K}_{T}, \vec{p}_{3}-x_{3} \vec{K}_{T}\right) \\
& +2 \delta^{a d} \delta^{b c} \psi^{*}\left(\vec{p}_{1}-\vec{q}_{1}-\vec{q}_{4}-x_{1} \vec{K}_{T}, \vec{p}_{2}-\vec{q}_{2}-\vec{q}_{3}-x_{2} \vec{K}_{T}, \vec{p}_{3}-x_{3} \vec{K}_{T}\right) \\
& -\delta^{a b} \delta^{c d} \psi^{*}\left(\vec{p}_{1}-\vec{q}_{1}-\vec{q}_{2}-\vec{q}_{3}-x_{1} \vec{K}_{T}, \vec{p}_{2}-\vec{q}_{4}-x_{2} \vec{K}_{T}, \vec{p}_{3}-x_{3} \vec{K}_{T}\right) \\
& -\delta^{a b} \delta^{c d} \psi^{*}\left(\vec{p}_{1}-\vec{q}_{1}-x_{1} \vec{K}_{T}, \vec{p}_{2}-\vec{q}_{2}-\vec{q}_{3}-\vec{q}_{4}-x_{2} \vec{K}_{T}, \vec{p}_{3}-x_{3} \vec{K}_{T}\right) \\
& -\delta^{a b} \delta^{c d} \psi^{*}\left(\vec{p}_{1}-\vec{q}_{1}-\vec{q}_{2}-\vec{q}_{4}-x_{1} \vec{K}_{T}, \vec{p}_{2}-\vec{q}_{3}-x_{2} \vec{K}_{T}, \vec{p}_{3}-x_{3} \vec{K}_{T}\right) \\
& -\delta^{a c} \delta^{d b} \psi^{*}\left(\vec{p}_{1}-\vec{q}_{1}-\vec{q}_{3}-\vec{q}_{4}-x_{1} \vec{K}_{T}, \vec{p}_{2}-\vec{q}_{2}-x_{2} \vec{K}_{T}, \vec{p}_{3}-x_{3} \vec{K}_{T}\right) \\
& -\delta^{a b} \delta^{c d} \psi^{*}\left(\vec{p}_{1}-\vec{q}_{1}-\vec{q}_{2}-x_{1} \vec{K}_{T}, \vec{p}_{2}-\vec{q}_{3}-x_{2} \vec{K}_{T}, \vec{p}_{3}-\vec{q}_{4}-x_{3} \vec{K}_{T}\right) \\
& -\delta^{a c} \delta^{b d} \psi^{*}\left(\vec{p}_{1}-\vec{q}_{1}-\vec{q}_{3}-x_{1} \vec{K}_{T}, \vec{p}_{2}-\vec{q}_{2}-x_{2} \vec{K}_{T}, \vec{p}_{3}-\vec{q}_{4}-x_{3} \vec{K}_{T}\right) \\
& -\delta^{a d} \delta^{b c} \psi^{*}\left(\vec{p}_{1}-\vec{q}_{1}-\vec{q}_{4}-x_{1} \vec{K}_{T}, \vec{p}_{2}-\vec{q}_{2}-x_{2} \vec{K}_{T}, \vec{p}_{3}-\vec{q}_{3}-x_{3} \vec{K}_{T}\right) \\
& -\delta^{a d} \delta^{b c} \psi^{*}\left(\vec{p}_{1}-\vec{q}_{1}-x_{1} \vec{K}_{T}, \vec{p}_{2}-\vec{q}_{2}-\vec{q}_{3}-x_{2} \vec{K}_{T}, \vec{p}_{3}-\vec{q}_{4}-x_{3} \vec{K}_{T}\right) \\
& -\delta^{a b} \delta^{c d} \psi^{*}\left(\vec{p}_{1}-\vec{q}_{1}-x_{1} \vec{K}_{T}, \vec{p}_{2}-\vec{q}_{2}-x_{2} \vec{K}_{T}, \vec{p}_{3}-\vec{q}_{3}-\vec{q}_{4}-x_{3} \vec{K}_{T}\right) \\
& \left.-\delta^{a c} \delta^{b d} \psi^{*}\left(\vec{p}_{1}-\vec{q}_{1}-x_{1} \vec{K}_{T}, \vec{p}_{2}-\vec{q}_{2}-\vec{q}_{4}-x_{2} \vec{K}_{T}, \vec{p}_{3}-\vec{q}_{3}-x_{3} \vec{K}_{T}\right)\right\} .
\end{aligned}
$$


The $C$-odd part of $\left\langle\rho^{4}\right\rangle$ is

$$
\begin{aligned}
& \left\langle\rho^{a}\left(\vec{q}_{1}\right) \rho^{b}\left(\vec{q}_{2}\right) \rho^{c}\left(\vec{q}_{3}\right) \rho^{d}\left(\vec{q}_{4}\right)\right\rangle_{f d}=\frac{i}{8} g^{4} \int \mathrm{d} x_{1} \mathrm{~d} x_{2} \mathrm{~d} x_{3} \delta\left(1-x_{1}-x_{2}-x_{3}\right) \int \frac{\mathrm{d}^{2} p_{1} \mathrm{~d}^{2} p_{2} \mathrm{~d}^{2} p_{3}}{\left(16 \pi^{3}\right)^{2}} \delta\left(\vec{p}_{1}+\vec{p}_{2}+\vec{p}_{3}\right) \psi\left(\vec{p}_{1}, \vec{p}_{2}, \vec{p}_{3}\right) \\
& \times\left\{\left(d^{\text {abe }} f^{c d e}+f^{\text {abe }} d^{\text {cde }}\right) \psi^{*}\left(\vec{p}_{1}-\vec{q}_{1}-\vec{q}_{2}-\vec{q}_{3}-\vec{q}_{4}-x_{1} \vec{K}_{T}, \vec{p}_{2}-x_{2} \vec{K}_{T}, \vec{p}_{3}-x_{3} \vec{K}_{T}\right)\right. \\
& -\left(d^{a b e} f^{c d e}+f^{a b e} d^{c d e}\right) \psi^{*}\left(\vec{p}_{1}-\vec{q}_{1}-\vec{q}_{2}-x_{1} \vec{K}_{T}, \vec{p}_{2}-\vec{q}_{3}-\vec{q}_{4}-x_{2} \vec{K}_{T}, \vec{p}_{3}-x_{3} \vec{K}_{T}\right) \\
& -\left(d^{\text {ace }} f^{b d e}+f^{a c e} d^{\text {bde }}\right) \psi^{*}\left(\vec{p}_{1}-\vec{q}_{1}-\vec{q}_{3}-x_{1} \vec{K}_{T}, \vec{p}_{2}-\vec{q}_{2}-\vec{q}_{4}-x_{2} \vec{K}_{T}, \vec{p}_{3}-x_{3} \vec{K}_{T}\right) \\
& -\left(d^{\text {ade }} f^{b c e}+f^{\text {ade }} d^{\text {bce }}\right) \psi^{*}\left(\vec{p}_{1}-\vec{q}_{1}-\vec{q}_{4}-x_{1} \vec{K}_{T}, \vec{p}_{2}-\vec{q}_{2}-\vec{q}_{3}-x_{2} \vec{K}_{T}, \vec{p}_{3}-x_{3} \vec{K}_{T}\right) \\
& -\left(d^{\text {abe }} f^{c d e}+f^{a b e} d^{c d e}\right) \psi^{*}\left(\vec{p}_{1}-\vec{q}_{1}-\vec{q}_{2}-\vec{q}_{3}-x_{1} \vec{K}_{T}, \vec{p}_{2}-\vec{q}_{4}-x_{2} \vec{K}_{T}, \vec{p}_{3}-x_{3} \vec{K}_{T}\right) \\
& -\left(d^{\text {abe }} f^{c d e}+f^{\text {abe }} d^{\text {cde }}\right) \psi^{*}\left(\vec{p}_{1}-\vec{q}_{1}-x_{1} \vec{K}_{T}, \vec{p}_{2}-\vec{q}_{2}-\vec{q}_{3}-\vec{q}_{4}-x_{2} \vec{K}_{T}, \vec{p}_{3}-x_{3} \vec{K}_{T}\right) \\
& -\left(d^{a b e} f^{d c e}+f^{a b e} d^{d c e}\right) \psi^{*}\left(\vec{p}_{1}-\vec{q}_{1}-\vec{q}_{2}-\vec{q}_{4}-x_{1} \vec{K}_{T}, \vec{p}_{2}-\vec{q}_{3}-x_{2} \vec{K}_{T}, \vec{p}_{3}-x_{3} \vec{K}_{T}\right) \\
& -\left(d^{a c e} f^{d b e}+f^{a c e} d^{d b e}\right) \psi^{*}\left(\vec{p}_{1}-\vec{q}_{1}-\vec{q}_{3}-\vec{q}_{4}-x_{1} \vec{K}_{T}, \vec{p}_{2}-\vec{q}_{2}-x_{2} \vec{K}_{T}, \vec{p}_{3}-x_{3} \vec{K}_{T}\right) \\
& +2 f^{\text {abe }} d^{\text {cde }} \psi^{*}\left(\vec{p}_{1}-\vec{q}_{1}-\vec{q}_{2}-x_{1} \vec{K}_{T}, \vec{p}_{2}-\vec{q}_{3}-x_{2} \vec{K}_{T}, \vec{p}_{3}-\vec{q}_{4}-x_{3} \vec{K}_{T}\right) \\
& +2 f^{a c e} d^{b d e} \psi^{*}\left(\vec{p}_{1}-\vec{q}_{1}-\vec{q}_{3}-x_{1} \vec{K}_{T}, \vec{p}_{2}-\vec{q}_{2}-x_{2} \vec{K}_{T}, \vec{p}_{3}-\vec{q}_{4}-x_{3} \vec{K}_{T}\right) \\
& +2 f^{\text {ade }} d^{\text {bce }} \psi^{*}\left(\vec{p}_{1}-\vec{q}_{1}-\vec{q}_{4}-x_{1} \vec{K}_{T}, \vec{p}_{2}-\vec{q}_{2}-x_{2} \vec{K}_{T}, \vec{p}_{3}-\vec{q}_{3}-x_{3} \vec{K}_{T}\right) \\
& +2 f^{\text {bce }} d^{\text {ade }} \psi^{*}\left(\vec{p}_{1}-\vec{q}_{1}-x_{1} \vec{K}_{T}, \vec{p}_{2}-\vec{q}_{2}-\vec{q}_{3}-x_{2} \vec{K}_{T}, \vec{p}_{3}-\vec{q}_{4}-x_{3} \vec{K}_{T}\right) \\
& +2 f^{c d e} d^{\text {abe }} \psi^{*}\left(\vec{p}_{1}-\vec{q}_{1}-x_{1} \vec{K}_{T}, \vec{p}_{2}-\vec{q}_{2}-x_{2} \vec{K}_{T}, \vec{p}_{3}-\vec{q}_{3}-\vec{q}_{4}-x_{3} \vec{K}_{T}\right) \\
& \left.+2 f^{\text {bde }} d^{\text {ace }} \psi^{*}\left(\vec{p}_{1}-\vec{q}_{1}-x_{1} \vec{K}_{T}, \vec{p}_{2}-\vec{q}_{2}-\vec{q}_{4}-x_{2} \vec{K}_{T}, \vec{p}_{3}-\vec{q}_{3}-x_{3} \vec{K}_{T}\right)\right\} \text {. }
\end{aligned}
$$

Using SU(3) identities, ${ }^{9}$ we verified that Eqs. (B14)-(B17) agree with the expressions in Sec. 4.4 of Ref. [46].

\section{APPENDIX C: WEIZSÄCKER-WILLIAMS GLUON DISTRIBUTION}

To leading order in $A^{+}$the field in L.C. gauge is given by $A^{i}(\vec{q})=-i q^{i} A^{+}(q)$. This leads to the WW gluon distributions

$$
\begin{aligned}
\delta^{i j}\left\langle A^{i a}(\vec{q}) A^{j a}(-\vec{q})\right\rangle & =\left(2 \frac{q^{i} q^{j}}{q^{2}}-\delta^{i j}\right)\left\langle A^{i a}(\vec{q}) A^{j a}(-\vec{q})\right\rangle \\
& =\frac{N_{c}^{2}-1}{2 q^{2}} g^{2} G_{2}(\vec{q},-\vec{q}) .
\end{aligned}
$$

At this order the conventional and linearly polarized gluon distributions are equal, and there is maximal polarization. Due to "color neutrality" of the proton, $G_{2}(\vec{q},-\vec{q}) / q^{2}$ does not diverge as $q \rightarrow 0$.

Solving Eq. (17) to quadratic order in $A^{+}$one has [50,51]

$$
\begin{aligned}
A^{i a}(\vec{q})= & -i q^{i} A^{+a}(\vec{q})+\frac{i g}{2} f^{a b c}\left(\frac{q^{i} q^{j}}{q^{2}}-\delta^{i j}\right) \\
& \times \int_{k} k^{j} A^{+b}(\vec{q}-\vec{k}) A^{+c}(\vec{k}) .
\end{aligned}
$$

\footnotetext{
${ }^{9}$ See Ref. [49], in particular Eq. (2.22).
}

This corresponds to the soft, "quasiclassical" field of recoil less valence quark sources. It is assumed that the contribution from diagrams corresponding to the internal exchange of a gluon over a large longitudinal distance $x^{-}$is suppressed; see the detailed discussion by Kovchegov in Ref. [51].

The contribution to $A^{i}$ at quadratic order in $A^{+}$leads to a correction to the WW gluon distributions at fourth order in $A^{+}$(fig. 9) [52]

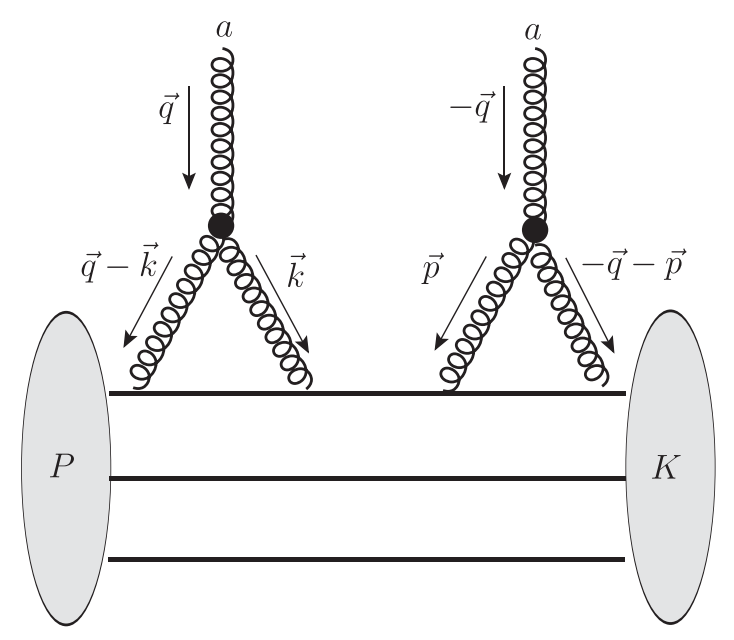

FIG. 9. One of the diagrams for the WW gluon distribution at fourth order in $g A^{+}$. 


$$
\begin{array}{r}
\Delta x G^{(1)}(x, \vec{q})=-\Delta x h_{\perp}^{(1)}(x, \vec{q})=\frac{1}{4 \pi^{3}} \frac{g^{2}}{4} f^{a b e} f^{c d e} \int_{k, p}\left(\frac{\vec{k} \cdot \vec{q} \vec{p} \cdot \vec{q}}{q^{2}}-\vec{k} \cdot \vec{p}\right)\left\langle A^{+a}(\vec{q}-\vec{k}) A^{+b}(\vec{k}) A^{+c}(-\vec{q}-\vec{p}) A^{+d}(\vec{p})\right\rangle \\
=\frac{1}{4 \pi^{3}} \frac{g^{2}}{4} f^{a b e} f^{c d e} \int_{k, p} \frac{1}{k^{2}} \frac{1}{p^{2}} \frac{1}{(\vec{q}-\vec{k})^{2}} \frac{1}{(\vec{q}+\vec{p})^{2}}\left(\frac{\vec{k} \cdot \vec{q} \vec{p} \cdot \vec{q}}{q^{2}}-\vec{k} \cdot \vec{p}\right)\left\langle\rho^{a}(\vec{q}-\vec{k}) \rho^{b}(\vec{k}) \rho^{c}(-\vec{q}-\vec{p}) \rho^{d}(\vec{p})\right\rangle .
\end{array}
$$

There is no contribution from cubic order in $A^{+}$as this is proportional to the product of the longitudinal L.C. gauge field $A^{i}$ at leading order with the transverse part of $A^{j}$ at quadratic order (or vice versa), contracted with either $\delta^{i j}$ or $\left(2 \frac{q^{i} q^{j}}{q^{2}}-\delta^{i j}\right)$, which gives zero. Note that the parentheses in Eqs. (C3) and (C4) can also be written in terms of the $2 \mathrm{~d}$ cross product as $[(\vec{q}-\vec{k}) \times \vec{q}][(\vec{q}+\vec{p}) \times \vec{q}] / q^{2}$.

With $\left\langle\rho^{4}\right\rangle$ from eq. (B13) and

$$
\begin{aligned}
& f(\vec{q})=\int \mathrm{d} x_{1} \mathrm{~d} x_{2} \mathrm{~d} x_{3} \delta\left(1-x_{1}-x_{2}-x_{3}\right) \int \frac{\mathrm{d}^{2} p_{1} \mathrm{~d}^{2} p_{2} \mathrm{~d}^{2} p_{3}}{\left(16 \pi^{3}\right)^{2}} \delta\left(\vec{p}_{1}+\vec{p}_{2}+\vec{p}_{3}\right) \psi^{*}\left(\vec{p}_{1}-\vec{q}, \vec{p}_{2}+\vec{q}, \vec{p}_{3}\right) \psi\left(\vec{p}_{1}, \vec{p}_{2}, \vec{p}_{3}\right), \\
& g\left(\vec{q}_{1}, \vec{q}_{2}\right)= \int \mathrm{d} x_{1} \mathrm{~d} x_{2} \mathrm{~d} x_{3} \delta\left(1-x_{1}-x_{2}-x_{3}\right) \int \frac{\mathrm{d}^{2} p_{1} \mathrm{~d}^{2} p_{2} \mathrm{~d}^{2} p_{3}}{\left(16 \pi^{3}\right)^{2}} \delta\left(\vec{p}_{1}+\vec{p}_{2}+\vec{p}_{3}\right) \\
& \times \psi^{*}\left(\vec{p}_{1}-\vec{q}_{1}, \vec{p}_{2}-\vec{q}_{2}, \vec{p}_{3}+\vec{q}_{1}+\vec{q}_{2}\right) \psi\left(\vec{p}_{1}, \vec{p}_{2}, \vec{p}_{3}\right),
\end{aligned}
$$

we can write the correction in the form

$$
\begin{aligned}
\Delta x G^{(1)}(x, \vec{q})= & -\Delta x h_{\perp}^{(1)}(x, \vec{q})=\frac{1}{4 \pi^{3}} \frac{3}{4} g^{6} \int_{k, p} \frac{1}{k^{2}} \frac{1}{p^{2}} \frac{1}{(\vec{q}-\vec{k})^{2}} \frac{1}{(\vec{q}+\vec{p})^{2}}\left(\frac{\vec{k} \cdot \vec{q} \vec{p} \cdot \vec{q}}{q^{2}}-\vec{k} \cdot \vec{p}\right) \\
& \times[-3+3 f(\vec{q})+2 f(\vec{p}+\vec{k})-2 f(\vec{p}+\vec{q}-\vec{k})+3 f(\vec{p})+3 f(\vec{q}-\vec{k})-3 f(\vec{p}+\vec{q})-3 f(\vec{k}) \\
& +g(\vec{k}, \vec{p})-g(\vec{p}+\vec{q}-\vec{k}, \vec{k})-g(\vec{q}-\vec{k}, \vec{p})+g(\vec{q}-\vec{k}, \vec{p}+\vec{k})] .
\end{aligned}
$$

The bracket vanishes if any two momenta $(\vec{p}, \vec{q}$ or $\vec{k}, \vec{q}$ or $\vec{p}, \vec{k})$ are taken to zero. At finite $\vec{q}$ the integral is free of infrared divergences and can be evaluated by Monte Carlo integration.

The correction at order $\left(A^{+}\right)^{4}$ increases with decreasing transverse momentum and eventually overwhelms the leading contribution $\sim\left(A^{+}\right)^{2}$. At such low $\vec{q}$ the result can no longer be trusted, and a resummation to all powers of $A^{+}$would be required. However, it is interesting to note that at very small $x$ some configurations of the proton correspond to negative $x h_{\perp}^{(1)}(x, q)$ at $q$ of order the saturation scale, even when the function is resummed to all orders in $A^{+}$[52].

If the four-charge correlator of Eq. (C4) is replaced by a sum over pairwise contractions,

$$
\begin{aligned}
\left\langle\rho^{a}(\vec{q}-\vec{k}) \rho^{b}(\vec{k}) \rho^{c}(-\vec{q}-\vec{p}) \rho^{d}(\vec{p})\right\rangle \rightarrow & \left\langle\rho^{a}(\vec{q}-\vec{k}) \rho^{b}(\vec{k})\right\rangle\left\langle\rho^{c}(-\vec{q}-\vec{p}) \rho^{d}(\vec{p})\right\rangle+\left\langle\rho^{a}(\vec{q}-\vec{k}) \rho^{c}(-\vec{q}-\vec{p})\right\rangle\left\langle\rho^{b}(\vec{k}) \rho^{d}(\vec{p})\right\rangle \\
& +\left\langle\rho^{a}(\vec{q}-\vec{k}) \rho^{d}(\vec{p})\right\rangle\left\langle\rho^{b}(\vec{k}) \rho^{c}(-\vec{q}-\vec{p})\right\rangle
\end{aligned}
$$

then the correction to the WW gluon distribution becomes

$$
\begin{aligned}
\left.\Delta x G^{(1)}(x, \vec{q})\right|_{\text {Gauss }} & =-\left.\Delta x h_{\perp}^{(1)}(x, \vec{q})\right|_{\text {Gauss }} \\
& =\frac{g^{6} N_{c}\left(N_{c}^{2}-1\right)}{32 \pi^{3}} \int_{k, p} \frac{1}{k^{2}} \frac{1}{p^{2}} \frac{1}{(\vec{q}-\vec{k})^{2}} \frac{1}{(\vec{q}+\vec{p})^{2}}\left(\frac{\vec{k} \cdot \vec{q} \vec{p} \cdot \vec{q}}{q^{2}}-\vec{k} \cdot \vec{p}\right) G_{2}(\vec{k}-\vec{q}, \vec{q}+\vec{p}) G_{2}(\vec{k}, \vec{p}) .
\end{aligned}
$$

Note that the $\left\langle\rho^{2}\right\rangle$ correlators in (C8) are nonforward matrix elements. The dominant contribution to the integral in Eq. (C9) is from $|\vec{k}+\vec{p}|$ on the order of the transverse momentum of the quarks in the proton so that both $G_{2}$ correlators are evaluated for small momentum transfer; their one-body GPD limit suffices for high $q$. 


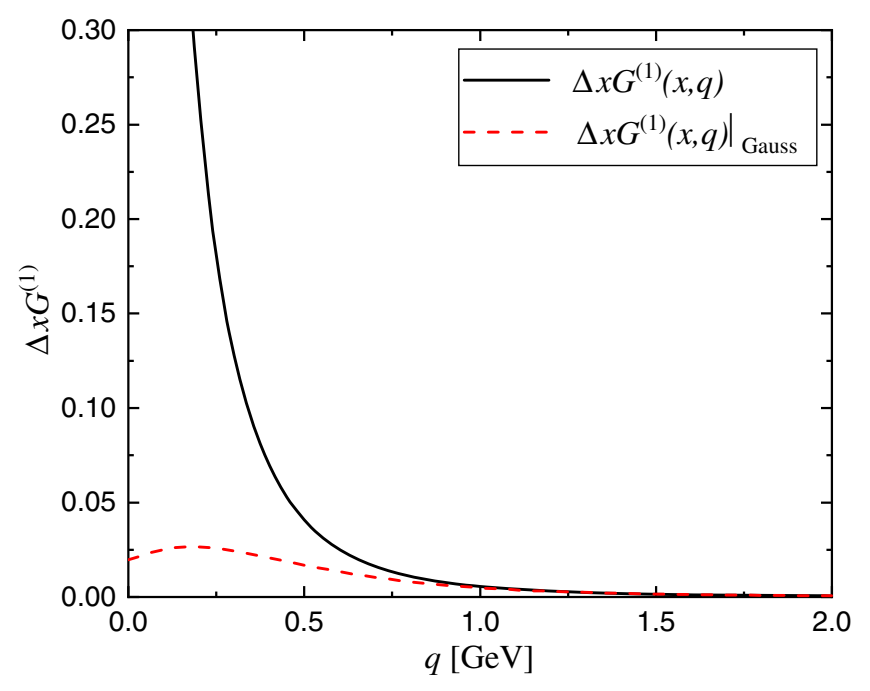

FIG. 10. The next-to-leading twist correction to the WW gluon distributions. The complete correlator of four $A^{+}$is compared to the sum over pairwise quadratic correlators.

Figure 10 shows a numerical comparison of Eq. (C9) to the complete result (C7). They agree at high transverse momentum where, however, the correction due to the transverse part of $A^{i a}$ is much smaller than the leading contribution. At $q \sim 0.2 \mathrm{GeV}$ the Gaussian approximation we described underestimates the true correction to the WW gluon distributions by about one order of magnitude.

Finally, we present expressions for the resummed form of $x G^{(1)}(x, \vec{q})$ and $x h_{\perp}^{(1)}(x, \vec{q})$ in a (large- $\left.N_{c}\right)$ Gaussian approximation for the general correlator $\left\langle\rho^{a}\left(\vec{q}_{1}\right) \rho^{b}\left(\vec{q}_{2}\right)\right\rangle=$ $\frac{1}{2} \delta^{a b} g^{2} G_{2}\left(\vec{q}_{1}, \vec{q}_{2}\right)$. Relaxing the assumption of translational invariance in the transverse plane, Eqs. (30) and (31) of Ref. [39] become

$$
\begin{aligned}
x h_{\perp}^{(1)}(x, \vec{q})= & \frac{N_{c}}{\alpha_{s}} \int \frac{\mathrm{d}^{2} r}{(2 \pi)^{2}} \int \frac{\mathrm{d}^{2} b}{(2 \pi)^{2}} e^{-i \vec{q} \cdot \vec{r}}\left(1-S^{2}\right) \\
& \times \frac{1}{\Gamma}\left(2\left(\hat{q} \cdot \vec{\nabla}_{r}\right)^{2}-\nabla_{r}^{2}\right) \Gamma \\
x G^{(1)}(x, \vec{q})= & \frac{N_{c}}{\alpha_{s}} \int \frac{\mathrm{d}^{2} r}{(2 \pi)^{2}} \int \frac{\mathrm{d}^{2} b}{(2 \pi)^{2}} e^{-i \vec{q} \cdot \vec{r}}\left(1-S^{2}\right) \frac{1}{\Gamma} \nabla_{r}^{2} \Gamma .
\end{aligned}
$$

Here,

$$
S(\vec{r}, \vec{b})=\exp \left(-\frac{1}{2} C_{F} \Gamma(\vec{r}, \vec{b})\right)
$$

denotes the dipole scattering matrix, and

$$
\Gamma(\vec{r}, \vec{b})=\left(4 \pi \alpha_{s}\right)^{2} \int_{p, q} \frac{e^{i(\vec{p}-\vec{q}) \cdot \vec{b}}}{p^{2} q^{2}}\left(1-e^{i(\vec{p}+\vec{q}) \cdot \vec{r}}\right) G_{2}(\vec{p},-\vec{q}) .
$$

The MV model correlator is recovered if one averages $\vec{b}$ over a large transverse area $S_{\perp}$ and replaces $G_{2}(\vec{p},-\vec{p})$ by a constant proportional to $\mu^{2} S_{\perp}$ (which also requires one to introduce an IR cutoff $\Lambda_{\mathrm{IR}}$ ).

We refrain from a numerical evaluation of Eqs. (C10)(C13) here which is rather tedious. Given that the saturation scale for nonlinear dynamics in the proton at $x \sim 0.1$ is rather small, we expect that for $q \gtrsim 0.5 \mathrm{GeV}$ the resummation does not give a significant correction to Eq. (C1) either.
[1] D. Boer et al., arXiv:1108.1713; A. Accardi et al., Eur. Phys. J. A 52, 268 (2016).

[2] L. D. McLerran and R. Venugopalan, Phys. Rev. D 49, 2233 (1994); 49, 3352 (1994); see, also, Yu. V. Kovchegov, Phys. Rev. D 54, 5463 (1996).

[3] A. Dumitru, G. A. Miller, and R. Venugopalan, Phys. Rev. D 98, 094004 (2018).

[4] I. Balitsky, Nucl. Phys. B463, 99 (1996); Y. V. Kovchegov, Phys. Rev. D 60, 034008 (1999).

[5] J. L. Albacete, N. Armesto, J. G. Milhano, and C. A. Salgado, Phys. Rev. D 80, 034031 (2009); J. L. Albacete, N. Armesto, J. G. Milhano, P. Quiroga-Arias, and C. A. Salgado, Eur. Phys. J. C 71, 1705 (2011).

[6] E. Iancu, J. D. Madrigal, A. H. Mueller, G. Soyez, and D. N. Triantafyllopoulos, Phys. Lett. B 750, 643 (2015);
B. Ducloué, E. Iancu, G. Soyez, and D. N. Triantafyllopoulos, arXiv:1912.09196.

[7] Y. V. Kovchegov, L. Szymanowski, and S. Wallon, Phys. Lett. B 586, 267 (2004); Y. Hatta, E. Iancu, K. Itakura, and L. McLerran, Nucl. Phys. A760, 172 (2005); T. Lappi, A. Ramnath, K. Rummukainen, and H. Weigert, Phys. Rev. D 94, 054014 (2016).

[8] X. Yao, Y. Hagiwara, and Y. Hatta, Phys. Lett. B 790, 361 (2019).

[9] P. J. Mulders and J. Rodrigues, Phys. Rev. D 63, 094021 (2001); C. J. Bomhof, P. J. Mulders, and F. Pijlman, Eur. Phys. J. C 47, 147 (2006); S. Meissner, A. Metz, and K. Goeke, Phys. Rev. D 76, 034002 (2007).

[10] D. Boer, P. J. Mulders, and C. Pisano, Phys. Rev. D 80, 094017 (2009); E. Akcakaya, A. Schäfer, and J. Zhou, Phys. 
Rev. D 87, 054010 (2013); C. Marquet, C. Roiesnel, and P. Taels, Phys. Rev. D 97, 014004 (2018).

[11] D. Boer, S. J. Brodsky, P. J. Mulders, and C. Pisano, Phys. Rev. Lett. 106, 132001 (2011); C. Pisano, D. Boer, S. J. Brodsky, M. G. A. Buffing, and P. J. Mulders, J. High Energy Phys. 10 (2013) 024; D. Boer, P. J. Mulders, C. Pisano, and J. Zhou, J. High Energy Phys. 08 (2016) 001; A. V. Efremov, N. Y. Ivanov, and O. V. Teryaev, Phys. Lett. B 777, 435 (2018); 780, 303 (2018).

[12] A. Dumitru, V. Skokov, and T. Ullrich, Phys. Rev. C 99, 015204 (2019); H. Mäntysaari, N. Mueller, F. Salazar, and B. Schenke, arXiv:1912.05586 [Phys. Rev. Lett. (to be published)].

[13] F. Dominguez, J. W. Qiu, B. W. Xiao, and F. Yuan, Phys. Rev. D 85, 045003 (2012).

[14] A. Dumitru, T. Lappi, and V. Skokov, Phys. Rev. Lett. 115, 252301 (2015).

[15] T. Altinoluk, R. Boussarie, C. Marquet, and P. Taels, arXiv:2001.00765.

[16] J. W. Qiu, M. Schlegel, and W. Vogelsang, Phys. Rev. Lett. 107, 062001 (2011).

[17] D. Boer, Few Body Syst. 58, 32 (2017); A. Mukherjee and S. Rajesh, Eur. Phys. J. C 77, 854 (2017); R. Kishore and A. Mukherjee, Phys. Rev. D 99, 054012 (2019).

[18] J. P. Lansberg, C. Pisano, F. Scarpa, and M. Schlegel, Phys. Lett. B 784, 217 (2018); 791, 420(E) (2019).

[19] J. P. Lansberg, C. Pisano, and M. Schlegel, Nucl. Phys. B920, 192 (2017).

[20] T. Lappi and S. Schlichting, Phys. Rev. D 97, 034034 (2018).

[21] G. P. Lepage and S. J. Brodsky, Phys. Rev. D 22, 2157 (1980); S. J. Brodsky, H. C. Pauli, and S. S. Pinsky, Phys. Rep. 301, 299 (1998).

[22] A. H. Mueller, arXiv:hep-ph/0111244.

[23] F. Dominguez, C. Marquet, B. W. Xiao, and F. Yuan, Phys. Rev. D 83, 105005 (2011).

[24] T. Altinoluk and R. Boussarie, J. High Energy Phys. 10 (2019) 208.

[25] J. Bartels and C. Ewerz, J. High Energy Phys. 09 (1999) 026.

[26] C. Ewerz, J. High Energy Phys. 04 (2001) 031.

[27] F. Schlumpf, Phys. Rev. D 47, 4114 (1993); 49, 6246(E) (1994); S. J. Brodsky and F. Schlumpf, Phys. Lett. B 329, 111 (1994).

[28] A. Donnachie and P. V. Landshoff, Z. Phys. C 2, 55 (1979); 2, 372(E) (1979); Phys. Lett. 123B, 345 (1983); Nucl. Phys. B231, 189 (1984).

[29] A. Dumitru and T. Stebel, Phys. Rev. D 99, 094038 (2019).

[30] F. E. Low, Phys. Rev. D 12, 163 (1975).
[31] Y. Hagiwara, Y. Hatta, B. W. Xiao, and F. Yuan, Phys. Lett. B 771, 374 (2017).

[32] K. J. Golec-Biernat and A. M. Stasto, Nucl. Phys. B668, 345 (2003); J. Berger and A. Stasto, Phys. Rev. D 83, 034015 (2011).

[33] R. Boussarie, Y. Hatta, L. Szymanowski, and S. Wallon, arXiv:1912.08182; also see J. Zhou, Phys. Rev. D 89, 074050 (2014).

[34] Y. V. Kovchegov and M. D. Sievert, Phys. Rev. D 86, 034028 (2012); 86, 079906(E) (2012).

[35] P. Hägler, B. Pire, L. Szymanowski, and O. V. Teryaev, Phys. Lett. B 535, 117 (2002); 540, 324(E) (2002); Eur. Phys. J. C 26, 261 (2002).

[36] D. Kharzeev, Y. V. Kovchegov, and K. Tuchin, Phys. Rev. D 68, 094013 (2003).

[37] R. Boussarie, Y. Hatta, B. W. Xiao, and F. Yuan, Phys. Rev. D 98, 074015 (2018).

[38] A. Metz and J. Zhou, Phys. Rev. D 84, 051503 (2011).

[39] A. Dumitru and V. Skokov, Phys. Rev. D 94, 014030 (2016).

[40] C. Marquet, E. Petreska, and C. Roiesnel, J. High Energy Phys. 10 (2016) 065.

[41] M. Diehl, Phys. Rep. 388, 41 (2003).

[42] H. Mäntysaari and B. Schenke, Phys. Rev. Lett. 117, 052301 (2016); Phys. Rev. D 94, 034042 (2016).

[43] S. Munier, A. M. Stasto, and A. H. Mueller, Nucl. Phys. B603, 427 (2001); H. Kowalski and D. Teaney, Phys. Rev. D 68, 114005 (2003).

[44] D. Binosi, J. Collins, C. Kaufhold, and L. Theussl, Comput. Phys. Commun. 180, 1709 (2009).

[45] M. R. Frank, B. K. Jennings, and G. A. Miller, Phys. Rev. C 54, 920 (1996); G. A. Miller, Phys. Rev. C 66, 032201 (2002); B. Pasquini and S. Boffi, Phys. Rev. D 76, 074011 (2007); B. Pasquini, S. Boffi, and P. Schweitzer, Mod. Phys. Lett. A 24, 2903 (2009); C. Lorcé, B. Pasquini, and M. Vanderhaeghen, J. High Energy Phys. 05 (2011) 041.

[46] J. Bartels and L. Motyka, Eur. Phys. J. C 55, 65 (2008).

[47] M. Fukugita and J. Kwiecinski, Phys. Lett. 83B, 119 (1979).

[48] J. Czyzewski, J. Kwiecinski, L. Motyka, and M. Sadzikowski, Phys. Lett. B 398, 400 (1997); 411, 402(E) (1997); R. Engel, D. Y. Ivanov, R. Kirschner, and L. Szymanowski, Eur. Phys. J. C 4, 93 (1998).

[49] A. J. MacFarlane, A. Sudbery, and P. H. Weisz, Commun. Math. Phys. 11, 77 (1968).

[50] A. Kovner, L. D. McLerran, and H. Weigert, Phys. Rev. D 52, 3809 (1995).

[51] Y. V. Kovchegov, Phys. Rev. D 55, 5445 (1997).

[52] A. Dumitru and V. Skokov, EPJ Web Conf. 172, 03009 (2018). 